TRANSACTIONS OF THE

AMERICAN MATHEMATICAL SOCIETY

Volume 352, Number 7, Pages 3211-3237

S 0002-9947(00)02610-6

Article electronically published on March 15, 2000

\title{
A FILTRATION OF SPECTRA ARISING FROM FAMILIES OF SUBGROUPS OF SYMMETRIC GROUPS
}

\author{
KATHRYN LESH
}

\begin{abstract}
Let $\mathcal{F}_{n}$ be a family of subgroups of $\Sigma_{n}$ which is closed under taking subgroups and conjugates. Such a family has a classifying space, $B \mathcal{F}_{n}$, and we showed in an earlier paper that a compatible choice of $\mathcal{F}_{n}$ for each $n$ gives a simplicial monoid $\coprod_{n} B \mathcal{F}_{n}$, which group completes to an infinite loop space. In this paper we define a filtration of the associated spectrum whose filtration quotients, given an extra condition on the families, can be identified in terms of the classifying spaces of the families of subgroups that were chosen. This gives a way to go from group theoretic data about the families to homotopy theoretic information about the associated spectrum. We calculate two examples. The first is related to elementary abelian $p$-groups, and the second gives a new expression for the desuspension of $S p^{m}\left(S^{0}\right) / S p^{m-1}\left(S^{0}\right)$ as a suspension spectrum.
\end{abstract}

\section{INTRODUCTION}

In this paper we explore the identification of infinite loop spaces that arise as group completions of certain classifying spaces with monoid structures. The prototypical example of such an infinite loop space is given by the Barratt-Priddy-Quillen theorem, which states that the group completion of the monoid $\amalg B \Sigma_{n}$ is the infinite loop space $Q S^{0}$. This theorem was generalized in $[\mathrm{L}$, where we replaced $B \Sigma_{n}$ with $B \mathcal{F}_{n}$, tom Dieck's classifying space of a family of subgroups of $\Sigma_{n}$, and then appealed to work of Segal to show that the resulting monoid $\amalg B \mathcal{F}_{n}$ has an infinite loop space as its group completion. The spaces $Q S^{0}$ and $K(\mathbf{Z}, 0)$ occur as extreme examples of the construction, but in general it is difficult to identify the infinite loop space, or the associated spectrum, that arises from a specific choice of families. In this paper we give a filtration of the associated spectrum such that the filtration quotients can be identified in terms of the classifying spaces of the families of subgroups that were chosen. This gives a way to take group theoretic data about the families of subgroups and obtain homotopy theoretic data about the spectrum associated to the resulting infinite loop space.

To describe our results in more detail, we require some notation. For each nonnegative integer $n$, let $\mathcal{F}_{n}$ be a family of subgroups of $\Sigma_{n}$, and let $\mathcal{F}$ denote the collection of families $\left\{\mathcal{F}_{n}\right\}_{n \in \mathbf{Z}_{+}}$. Let $B \mathcal{F}_{n}$ be the classifying space of $\mathcal{F}_{n}$, whose construction we review in Section 2. If the families are closed under block sum of permutations, then $\amalg B \mathcal{F}_{n}$ is a simplicial monoid, and we review in Section 2 how work of Segal applies to show that its group completion is an infinite loop space.

Received by the editors November 26, 1997.

2000 Mathematics Subject Classification. Primary 55P47; Secondary 55N20, 55P42.

(C)2000 American Mathematical Society 
The associated spectrum, which we denote by $A$, is natural in the sequence $\mathcal{F}$ of families. The goal is to identify $A$ in terms of the families that make up $\mathcal{F}$.

We define a filtration of $A$ by spectra $A_{m}$, called the complexity filtration of $A$. The filtration is defined by filtering the families, and thus is natural in $\mathcal{F}$. The main theorem of this paper is that, given an additional condition on the families, the product projection condition (see Definition ??), the filtration quotients $A_{m} / A_{m-1}$ can be naturally identified in terms of classifying spaces of families of subgroups.

Theorem 1.1. Let $\mathcal{F}$ be a compatible collection of families that satisfies the product projection condition. Let $A$ be the associated spectrum, and let $A_{m}$ denote the mth filtration of $A$ by complexity. Let $\mathcal{F}_{m}^{\prime}$ be the family of subgroups in $\mathcal{F}_{m}$ which do not act transitively on $\{1, \ldots, m\}$. Then there is a stable homotopy pushout diagram,

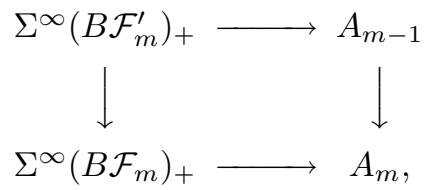

where the vertical maps are given by inclusions of the $(m-1)$ st complexity filtration in the mth, and the horizontal maps are given by mth family inclusion maps. The pushout diagram is natural in $\mathcal{F}$.

Since $A_{1}=S^{0}$ and hocolim ${ }_{m} A_{m} \simeq A$ (Proposition 2.9), we recover information about all of $A$ from the filtration.

We offer examples of the application of Theorem 1.1 in Section 7. First we show how the main example calculated in $[\mathrm{L}$, namely the spectrum associated to the elementary abelian $p$-groups with orbits of length at most $p$, can be calculated much more simply by the use of Theorem 1.1 Then we consider the family of all subgroups and show that the complexity filtration of the associated spectrum is the filtration of the integral Eilenberg-MacLane spectrum $H \mathbf{Z}$ by symmetric powers of spheres. Finally, we use Theorem 1.1 in conjunction with this calculation to show the following, and we recover some known results on symmetric powers of spheres.

Theorem 1.2. Let $\mathcal{F}_{m}^{\prime}$ denote the family of all subgroups of $\Sigma_{m}$ which do not act transitively on $\{1, \ldots, m\}$, and let $S p^{m}\left(S^{0}\right)$ denote the mth symmetric power of the sphere spectrum. Then

$$
S p^{m}\left(S^{0}\right) / S p^{m-1}\left(S^{0}\right) \simeq \Sigma \Sigma^{\infty} B \mathcal{F}_{m}^{\prime} .
$$

The rest of the paper is organized as follows. In Section 2, we review definitions and constructions from $[\mathrm{L}$, and we define the product projection condition and the complexity filtration, which appear in the statement of Theorem 1.1. In Section 3. we discuss the details of Segal's infinite loop space contruction as it applies to our situation, and we define the weight filtration, with which we later analyze the spaces that come out of Segal's construction. Section 4 is devoted to the proof of the main technical tool of the paper, Proposition 4.8, which identifies the homotopy type of the filtration quotients of the weight filtration in the case that the product projection condition is satisfied. In Section 5 , we construct the $m$ th family inclusion maps which appear as the horizontal maps in the pushout diagram of Theorem 1.1 and we show that in the case of stable homotopy they are related to the transfer. In Section [6] we give the proof of Theorem [1.1. Section 7 gives examples, and Theorem 1.2 is proved in Section 7.2 . 


\section{BACKGROUND AND DEFINITIONS}

In this section we give background information on how families of subgroups of symmetric groups lead to infinite loop spaces, and we define the terms that are needed for Theorem 1.1. We first collect definitions and propositions relating to classifying spaces of families of subgroups. Then we review from $[\mathrm{L}$ the symmetric monoidal category which leads from families of subgroups to infinite loop spaces. Next we define the product projection condition and the complexity filtration, and explain how they interact. Finally, we prove that hocolim $A_{m} \simeq A$.

In the following series of definitions and propositions, we collect the facts we will need about families of subgroups and their classifying spaces. Let $Y^{H}$ denote the fixed point set of the action of a group $H$ on a simplicial set $Y$.

\section{Definition 2.1.}

1. tD Let $G$ be a group and let $\mathcal{F}$ be a collection of subgroups of $G$. We call $\mathcal{F}$ a family if

(a) $H \in \mathcal{F}, \sigma \in G \Rightarrow \sigma H \sigma^{-1} \in \mathcal{F}$; and

(b) $H \in \mathcal{F}$ and $K$ a subgroup of $H \Rightarrow K \in \mathcal{F}$.

2. If $\mathcal{F}$ is a family of subgroups of $G$, let $\mathcal{E}_{\mathcal{F}}$ be the category whose objects are pairs $(O, x)$ where $O$ is a $G$-orbit with isotropy in $\mathcal{F}$ and $x \in O$. Morphisms $(O, x) \rightarrow\left(O^{\prime}, x^{\prime}\right)$ are $G$-equivariant maps $O \rightarrow O^{\prime}$ such that $x \mapsto x^{\prime}$.

3. If $\mathcal{F}$ is a family of subgroups of $G$, let $\mathcal{O}_{\mathcal{F}}$ be the category whose objects are $G$-orbits $O$ with isotropy in $\mathcal{F}$. Morphisms $O \rightarrow O^{\prime}$ are $G$-equivariant maps.

\section{Proposition 2.2.}

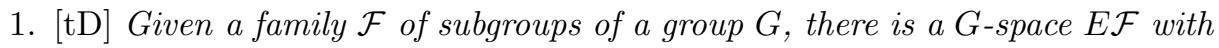
the property that

$$
\begin{aligned}
& (E \mathcal{F})^{H} \simeq * \quad \text { for } H \in \mathcal{F}, \\
& (E \mathcal{F})^{H}=\emptyset \quad \text { for } H \notin \mathcal{F} .
\end{aligned}
$$

The space EF is terminal up to homotopy among $G$-spaces whose isotropy groups lie in $\mathcal{F}$, and is known as the $\mathcal{F}$-universal $G$-space.

2. [E] The space $E \mathcal{F}$ is $G$-equivariantly homotopy equivalent to the nerve of $\mathcal{E}_{\mathcal{F}}$, where the $G$-action on $\mathcal{E}_{\mathcal{F}}$ comes from the action of $G$ on the distinguished point.

Definition 2.3. The classifying space of the family $\mathcal{F}$, written $B \mathcal{F}$, is the orbit space of the $G$-action on $E \mathcal{F}$.

Proposition 2.4. E The nerve of $\mathcal{O}_{\mathcal{F}}$ is homotopy equivalent to $B \mathcal{F}$.

The space $E \mathcal{F}$ should be regarded as a generalization of the free contractible $G$-space $E G$, which is recovered in the case that $\mathcal{F}$ consists of only the trivial subgroup. Similarly, $B \mathcal{F}$ generalizes the classifying space $B G$.

Next we turn to a description of how families of subgroups lead to infinite loop spaces. In particular, we show how to construct a symmetric monoidal category whose nerve is the simplicial monoid $\amalg B \mathcal{F}_{n}$ referred to in the introduction. By work of Segal (see $[\underline{\mathrm{S}}]$; also $[\mathrm{M}]$ and $[\overline{\mathrm{B}-\mathrm{F}}]$ ), the group completion of the nerve is an infinite loop space.

For each $n$, choose a family $\mathcal{F}_{n}$ of subgroups of $\Sigma_{n}$, and let $\mathcal{F}$ denote the collection of families $\left\{\mathcal{F}_{n}\right\}_{n \in \mathbf{Z}_{+}}$. 
Definition 2.5. Let $i_{1}, \ldots, i_{k}$ be nonnegative integers. The product family $\mathcal{F}_{i_{1}} \times$ $\ldots \times \mathcal{F}_{i_{k}}$ of subgroups of $\Sigma_{i_{1}} \times \ldots \times \Sigma_{i_{k}}$ is the family whose elements are either products $H_{1} \times \ldots \times H_{k}$ where each $H_{j} \in \mathcal{F}_{i_{j}}$, or subgroups of such products.

(Note the abuse of notation in that some elements of $\mathcal{F}_{i_{1}} \times \ldots \times \mathcal{F}_{i_{k}}$ may not actually be products of subgroups.)

Given nonnegative integers $i_{1}, \ldots, i_{k}$, let $n=i_{1}+\ldots+i_{k}$. We regard $\Sigma_{i_{1}} \times \ldots \times \Sigma_{i_{k}}$ as a subgroup of $\Sigma_{n}$ by letting $\Sigma_{i_{1}}$ act on $1,2, \ldots, i_{1}$, letting $\Sigma_{i_{2}}$ act on $i_{1}+1, \ldots, i_{1}+i_{2}$, and so on.

Definition 2.6. $\mathcal{F}$ is compatible if for all nonnegative integers $i_{1}, \ldots, i_{k}$,

$$
H \in \mathcal{F}_{i_{1}} \times \ldots \times \mathcal{F}_{i_{k}} \Rightarrow H \in \mathcal{F}_{n} .
$$

To obtain a symmetric monoidal category, recall from Definition 2.1 that $O_{\mathcal{F}_{n}}$ is the category of $\Sigma_{n}$-orbits with isotropy in $\mathcal{F}_{n}$ and equivariant maps. Let $\mathcal{O}_{\mathcal{F}}$ be the category which is the disjoint union of the categories $O_{\mathcal{F}_{n}}$. (Hence $\mathcal{O}_{\mathcal{F}}$ has one component for each nonnegative integer.) If $\mathcal{F}$ is compatible, then we can make $\mathcal{O}_{\mathcal{F}}$ into a symmetric monoidal category by defining the following bifunctor on it. Let $O_{1}$ be a $\Sigma_{i_{1}}$-orbit and let $O_{2}$ be a $\Sigma_{i_{2}}$-orbit. We define

$$
O_{1} \otimes O_{2}=\Sigma_{i_{1}+i_{2}} \times\left(\Sigma_{i_{1}} \times \Sigma_{i_{2}}\right)\left(O_{1} \times O_{2}\right) .
$$

Here the action of $\Sigma_{i_{1}} \times \Sigma_{i_{2}}$ on $\Sigma_{i_{1}+i_{2}}$ is through the inclusion $\Sigma_{i_{1}} \times \Sigma_{i_{2}} \hookrightarrow \Sigma_{i_{1}+i_{2}}$. Compatibility of $\mathcal{F}$ is required to guarantee that $O_{1} \otimes O_{2}$ has isotropy in $\mathcal{F}_{i_{1}+i_{2}}$ and hence is an orbit in $\mathcal{O}_{\mathcal{F}}$.

In later sections, we will need to use the commutativity isomorphisms for $\mathcal{O}_{\mathcal{F}}$ explicitly, so we digress to mention them here. If $O_{1}$ is a $\Sigma_{i_{1}}$-orbit, $O_{2}$ is a $\Sigma_{i_{2}}$-orbit, and $n=i_{1}+i_{2}$, we must give a natural isomorphism

$$
c: \Sigma_{n} \times_{\left(\Sigma_{i_{1}} \times \Sigma_{i_{2}}\right)}\left(O_{1} \times O_{2}\right) \rightarrow \Sigma_{n} \times_{\left(\Sigma_{i_{2}} \times \Sigma_{i_{1}}\right)}\left(O_{2} \times O_{1}\right) .
$$

Let $\tau \in \Sigma_{n}$ transpose the blocks $\left\{1, \ldots, i_{1}\right\}$ and $\left\{i_{1}+1, \ldots, i_{1}+i_{2}\right\}$. Then the commutativity isomorphism is defined by

$$
c\left(g,\left(x_{1}, x_{2}\right)\right)=\left(g \tau,\left(x_{2}, x_{1}\right)\right) .
$$

Returning to the construction of infinite loop spaces, we see that any compatible choice of families leads to a symmetric monoidal category $\mathcal{O}_{\mathcal{F}}$, and by $[\mathrm{S}]$ the nerve of this category, $\coprod B \mathcal{F}_{n}$, group completes to an infinite loop space. If each $\mathcal{F}_{n}$ consists only of the trivial subgroup, then $B \mathcal{F}_{n} \simeq B \Sigma_{n}$, and the Barratt-PriddyQuillen theorem tells us that $\coprod B \mathcal{F}_{n}$ group completes to $Q S^{0}$. If each $\mathcal{F}_{n}$ consists of all subgroups of $\Sigma_{n}$, then $B \mathcal{F}_{n} \simeq *$ and $\coprod B \mathcal{F}_{n}$ group completes to $\mathbf{Z}$, or $K(\mathbf{Z}, 0)$. Hence if $\coprod B \mathcal{F}_{n}$ has associated spectrum $A$, naturality in the choice of families gives maps $S^{0} \rightarrow A \rightarrow H \mathbf{Z}$ whose composite is the Hurewicz map. In other words, the spectra obtained by this construction interpolate between stable homotopy and integral homology.

Next we define the complexity filtration and the product projection condition, which appear in the statement of Theorem 1.1.

Definition 2.7. Let $H$ be a subgroup of $\Sigma_{n}$. We say that $H$ has complexity less than or equal to $m$ if the action of $H$ on the set $\{1, \ldots, n\}$ divides that set into orbits of length less than or equal to $m$. The $m$ th complexity filtration of a family $\mathcal{F}_{n}$, denoted $C_{m} \mathcal{F}_{n}$, is the subset of $\mathcal{F}_{n}$ consisting of subgroups of complexity less than or equal to $m$. 
We write $C_{m} \mathcal{F}$ for the collection $\left\{C_{m} \mathcal{F}_{n}\right\}_{n \in \mathbf{Z}_{+}}$. If $\mathcal{F}$ is a compatible collection, then $C_{m} \mathcal{F}$ is compatible as well.

Definition 2.8. Let $\mathcal{F}$ be a compatible collection of families with associated spectrum $A$. The spectrum associated to the collection $C_{m} \mathcal{F}$ is denoted $A_{m}$ and is called the $m$ th filtration of $A$ by complexity

The complexity filtration gives us information about all of the spectrum $A$, in the sense that the smallest filtration is $A_{1}=S^{0}$, and the direct limit of the filtrations is $A$ itself.

Proposition 2.9. With $A$ and $A_{m}$ as above, $A_{1}=S^{0}$ and

$$
\operatorname{hocolim}_{m} A_{m} \simeq A \text {. }
$$

Proof. The Barratt-Priddy-Quillen theorem says precisely that $A_{1}=S^{0}$, because the only subgroup of complexity 1 is the trivial subgroup.

Because $C_{m} \mathcal{F}_{n}=\mathcal{F}_{n}$ if $m \geq n$, we know that hocolim $m B C_{m} \mathcal{F}_{n} \simeq B \mathcal{F}_{n}$ and hence hocolim $_{m} \coprod_{n} B C_{m} \mathcal{F}_{n}=\coprod_{n} B \mathcal{F}_{n}$. To group complete a simplicial monoid, we compute $\Omega B(-)$, and this functor commutes with the homotopy colimit in question. (See, for example, [A, Chapter 3].) Thus the infinite loop space arising from the family $\mathcal{F}$ is the homotopy colimit of those arising from the families $C_{m} \mathcal{F}$, and the same is true for the associated spectra, that is, $\operatorname{hocolim}_{m} A_{m} \simeq A$.

The other condition on $\mathcal{F}$ which appears in the statement of Theorem 1.1 is the product projection condition, which is a converse to the compatibility condition.

Definition 2.10. $\mathcal{F}$ satisfies the product projection condition if for any nonnegative integers $i_{1}, \ldots, i_{k}$ and $n=i_{1}+\ldots+i_{k}$, we have that $H \in \mathcal{F}_{n}$ and $H \subseteq$ $\Sigma_{i_{1}} \times \ldots \times \Sigma_{i_{k}}$ imply $H \in \mathcal{F}_{i_{1}} \times \ldots \times \mathcal{F}_{i_{k}}$.

The geometric implications of this condition are discussed in Section 3 It is easy to check that the condition can be equivalently phrased by requiring that if $H \in \mathcal{F}_{n}$ and $H \subseteq \Sigma_{i_{1}} \times \ldots \times \Sigma_{i_{k}}$, then the projection of $H$ to each $\Sigma_{i_{j}}$ is in $\mathcal{F}_{i_{j}}$.

\section{Examples 2.11.}

1. Let $\mathcal{F}$ consist of all elementary abelian $p$-subgroups. Since homomorphic images of elementary abelian $p$-groups are again elementary abelian $p$-groups, $\mathcal{F}$ satisfies the product projection condition.

2. Let $\mathcal{F}$ consist of all subgroups of the alternating groups. Certainly $\mathcal{F}$ is compatible, because the product of two even permutations is again even. However, $\mathcal{F}$ does not satisfy the product projection condition, because an even permutation can be the product of two odd ones, and hence the projection of an even permutation to a smaller symmetric group can be odd.

Finally, we discuss the interaction of the complexity filtration and the product projection condition. Note that for any $m, C_{m-1} \mathcal{F}_{m}$ consists of the nontransitive subgroups in $\mathcal{F}_{m}$, or, in the language of Theorem 1.1. $C_{m-1} \mathcal{F}_{m}=\mathcal{F}_{m}^{\prime}$. According to Proposition 2.12 below, when we have the product projection condition, $\mathcal{F}_{m}^{\prime}$ consists of all elements of $\mathcal{F}_{m}$ which are forced on us by the families $\mathcal{F}_{1}, \ldots, \mathcal{F}_{m-1}$ 
under the compatibility condition. Hence the pushout diagram of Theorem [1.1]

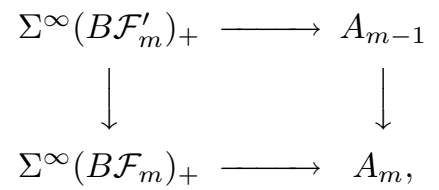

can be interpreted as saying that the difference between the spectra $A_{m}$ and $A_{m-1}$ is measured by the difference between the family $\mathcal{F}_{m}$ and the subfamily $\mathcal{F}_{m}^{\prime}$ of subgroups which are forced under compatibility by the previous choices $\mathcal{F}_{1}, \ldots, \mathcal{F}_{m-1}$. Said another way, the difference between $A_{m}$ and $A_{m-1}$ is caused by the genuinely new subgroups that are introduced in $\mathcal{F}_{m}$.

Proposition 2.12. Let $\mathcal{F}$ be a compatible collection of families satisfying the product projection condition and let $H \in \mathcal{F}_{m}^{\prime}$. Then there exist positive integers $i_{1}, \ldots, i_{k}$ with $i_{1}+\ldots+i_{k}=m$ such that $H \in \mathcal{F}_{i_{1}} \times \ldots \times \mathcal{F}_{i_{k}}$.

Proof. If $H \in \mathcal{F}_{m}^{\prime}=C_{m-1} \mathcal{F}_{m}$, then $H$ has orbits on $\{1, \ldots, m\}$ which are of length less than or equal to $m-1$, and hence

$$
H \subseteq \Sigma_{i_{1}} \times \ldots \times \Sigma_{i_{k}} \subseteq \Sigma_{m}
$$

for some $0<i_{1}, \ldots, i_{k} \leq m-1$ with $i_{1}+\ldots+i_{k}=m$. But then by the product projection condition, $H \in \mathcal{F}_{i_{1}} \times \ldots \times \mathcal{F}_{i_{k}}$.

\section{Segal's T-Category Construction as APplied to Families}

In order to prove Theorem 1.1 we will need to make a detailed analysis of Segal's $\Gamma$-space construction. Let $\mathcal{F}$ be a choice of compatible families, let $\mathcal{O}_{\mathcal{F}}$ be the associated symmetric monoidal category described in Section 2 , and let $\mathcal{O}_{\mathcal{F}}(X)$ be Segal's $\Gamma$-space construction applied to a simplicial set $X$. In this section, we review Segal's construction of $\mathcal{O}_{\mathcal{F}}(X)$ and we define a filtration on it called the weight filtration, which is the essential tool in the proof of Theorem 1.1. The weight filtration is analogous to the natural filtration of the Barratt-Eccles construction, which we describe in order to give a feel for the weight filtration's geometric meaning and to explain the role of the product projection condition. Finally we give an expression for the filtration quotients as the nerve of a category, which will be useful in constructing weak equivalences involving the filtration quotients and the nerves of other categories.

In [S], Segal defines a $\Gamma$-space as a functor $A$ from the category of finite pointed sets to the category of spaces, with the property that the pinch maps induce weak equivalences $A\left(S_{1} \vee S_{2}\right) \rightarrow A\left(S_{1}\right) \times A\left(S_{2}\right)$. Such a functor has the property that $A\left(S^{0}\right)$ group completes to an infinite loop space. A $\Gamma$-category is a functor $\mathcal{C}$ from pointed sets to categories with the property that pinch maps induce equivalences of categories $\mathcal{C}\left(S_{1} \vee S_{2}\right) \rightarrow \mathcal{C}\left(S_{1}\right) \times \mathcal{C}\left(S_{2}\right)$. Following a $\Gamma$-category $\mathcal{C}$ with the classifying space functor gives a $\Gamma$-space $|\mathcal{C}|$. Hence, if $\mathcal{C}$ is a $\Gamma$-category, then $|\mathcal{C}|$ is a $\Gamma$-space, and $\left|\mathcal{C}\left(S^{0}\right)\right|$ group completes to an infinite loop space. Finally, examples of $\Gamma$-categories arise from symmetric monoidal categories as detailed below. We will be applying this technique to the symmetric monoidal category $\mathcal{O}_{\mathcal{F}}$.

Let $\mathcal{C}$ be a symmetric monoidal category with symmetric monoidal product $\otimes$, and let $S$ be a finite pointed set. As we will need to use the details of Segal's construction, we will be very explicit about how to associate to $\mathcal{C}$ a $\Gamma$-category $S \rightarrow \mathcal{C}(S)$. Let $P(S)$ denote the set of (pointed) subsets of $S$. Call two pointed 
subsets of $S$ disjoint if the only element they have in common is the basepoint. An object in the category $\mathcal{C}(S)$ consists of the following data:

1. A function $f: P(S) \rightarrow \operatorname{Obj}(\mathcal{C})$;

2. For every $S_{1}, S_{2}$ disjoint elements of $P(S)$, an isomorphism $\alpha_{S_{1}, S_{2}}: f\left(S_{1}\right) \otimes$ $f\left(S_{2}\right) \rightarrow f\left(S_{1} \vee S_{2}\right)$. The collection of isomorphisms, which we call $\alpha_{*}$, must be coherent in the sense that the following diagrams commute for all choices of disjoint pointed subsets of $S$. (In each case the top horizontal map is given by the structure of $\mathcal{C}$ as a symmetric monoidal category.)

(a) Associativity:

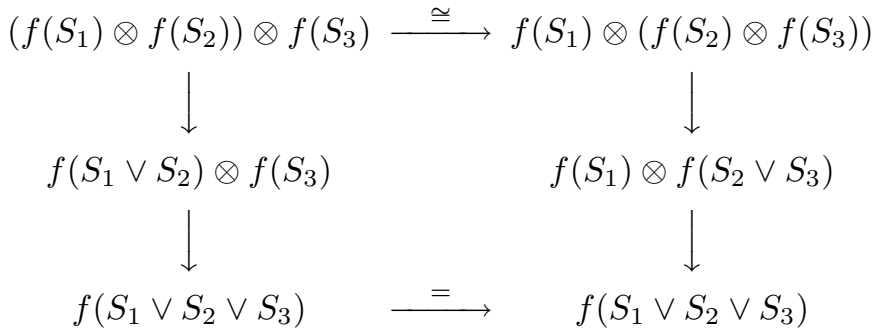

(b) Commutativity:

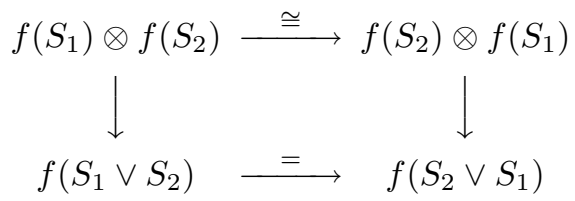

A morphism $\left(f_{1},\left(\alpha_{1}\right)_{*}\right) \rightarrow\left(f_{2},\left(\alpha_{2}\right)_{*}\right)$ in $\mathcal{C}(S)$ is a collection of morphisms $f_{1}\left(S^{\prime}\right) \rightarrow$ $f_{2}\left(S^{\prime}\right)$ in $\mathcal{C}$, one for each pointed subset $S^{\prime} \subseteq S$, which commute with the isomorphisms of $\left(\alpha_{1}\right)_{*}$ and $\left(\alpha_{2}\right)_{*}$.

If $h: S \rightarrow T$ is a map of finite pointed sets, we will also need information about the natural transformation $\mathcal{C}(h): \mathcal{C}(S) \rightarrow \mathcal{C}(T)$ that it induces. In particular, if $\mathcal{C}(h):\left(f, \alpha_{*}\right) \mapsto\left(g, \beta_{*}\right)$, we will need to know the function $g: P(T) \rightarrow \mathcal{C}$ in terms of $f: P(S) \rightarrow \mathcal{C}$. For a pointed subset $T^{\prime} \subseteq T$, let $h_{+}^{-1} T^{\prime}$ denote the subset of $S$ consisting of the basepoint and of elements of $S$ which map to nonbasepoint elements of $T^{\prime}$. Then to define the function $g: P(T) \rightarrow \mathcal{C}$, we take $g\left(T^{\prime}\right)=$ $f\left(h_{+}^{-1} T^{\prime}\right)$. The point of the definition of $h_{+}^{-1}$ is to exclude from the preimage of $T^{\prime}$ the nonbasepoint elements of $S$ which map to the basepoint of $T$. Otherwise, for example, preimages of disjoint pointed subsets of $T$ might not be disjoint pointed subsets of $S$, which would give trouble in defining the isomorphisms in $\beta_{*}$.

Now we give some definitions and lemmas on the application of Segal's work to our situation. Later in the section we give them some context and geometric interpretation by reviewing the construction of Barratt and Eccles for $Q X$ and explaining the analogies with our constructions. Let $S$ be a finite pointed set and fix an enumeration of the non-basepoint elements, $S=\left\{s_{1}, \ldots, s_{k}\right\}$. When we specify a subset of $S$ by listing its elements, the basepoint will be understood to be present, even though it is not specifically listed. For example, we write $\left\{s_{j}\right\}$ for the subset of $S$ consisting of the basepoint and the element $s_{j}$. Let $\mathcal{F}$ denote a compatible collection of families $\left\{\mathcal{F}_{n}\right\}_{n \in \mathbf{Z}_{+}}$and consider the $\Gamma$-category $S \mapsto \mathcal{O}_{\mathcal{F}}(S)$.

Definition 3.1. An object $\left(f, \alpha_{*}\right)$ of $\mathcal{O}_{\mathcal{F}}(S)$ has total weight $n$ if $f(S)$ is a $\Sigma_{n^{-}}$ orbit, and $\left(f, \alpha_{*}\right)$ has type $\left(i_{1}, \ldots, i_{k}\right)$ if $f\left(\left\{s_{j}\right\}\right)$ is a $\Sigma_{i_{j}}$-orbit for each $j$. 
Note that if $\left(f, \alpha_{*}\right)$ has total weight $n$ and type $\left(i_{1}, \ldots, i_{k}\right)$, then $n=i_{1}+\ldots+i_{k}$. This is because $f(S)$ is isomorphic to $f\left(\left\{s_{1}\right\}\right) \otimes \ldots \otimes f\left(\left\{s_{k}\right\}\right)$, which is a $\Sigma_{i_{1}+\ldots+i_{k}}$ orbit.

Lemma 3.2. The full subcategory of $\mathcal{O}_{\mathcal{F}}(S)$ of objects of type $\left(i_{1}, \ldots, i_{k}\right)$ is a component of $\mathcal{O}_{\mathcal{F}}(S)$.

Proof. First we claim that if objects have different types, then they are in different components. For if $\left(f, \alpha_{*}\right)$ and $\left(f^{\prime}, \alpha_{*}^{\prime}\right)$ have different types $\left(i_{1}, \ldots, i_{k}\right)$ and $\left(i_{1}^{\prime}, \ldots, i_{k}^{\prime}\right)$, then for some $j$, we have $i_{j} \neq i_{j}^{\prime}$. However, having a morphism $\left(f, \alpha_{*}\right) \rightarrow\left(f^{\prime}, \alpha_{*}^{\prime}\right)$ would require having a morphism $f\left(\left\{s_{j}\right\}\right) \rightarrow f^{\prime}\left(\left\{s_{j}\right\}\right)$ in $\mathcal{O}_{\mathcal{F}}$, and there are no such morphisms if $i_{j} \neq i_{j}^{\prime}$.

To show that the objects of type $\left(i_{1}, \ldots, i_{k}\right)$ form a single component of $\mathcal{O}_{\mathcal{F}}(S)$, we note that there is a (nonunique) object $\left(f, \alpha_{*}\right)$ of type $\left(i_{1}, \ldots, i_{k}\right)$ such that for every $S^{\prime} \in P(S)$, the orbit $f\left(S^{\prime}\right)$ is free, and there is a (nonunique) morphism from $\left(f, \alpha_{*}\right)$ to any other object of $\mathcal{O}_{\mathcal{F}}(S)$ with the same type.

We filter $\mathcal{O}_{\mathcal{F}}(S)$ by letting $W_{n} \mathcal{O}_{\mathcal{F}}(S)$ be the full subcategory of objects whose total weight is less than or equal to $n$, and we write $W_{n} / W_{n-1} \mathcal{O}_{\mathcal{F}}(S)$ for the $n$th filtration quotient. This filtration is critical in the proof of Theorem 1.1. We will need to know that the filtration is respected by maps of finite pointed sets. We prove the following more precise result, which is required in Section 4.

Lemma 3.3. Let $h: S \rightarrow T$ be a map of finite pointed sets. Let $\left(f, \alpha_{*}\right)$ be an object of $\mathcal{O}_{\mathcal{F}}(S)$ of type $\left(i_{1}, \ldots, i_{k}\right)$, and suppose that $\mathcal{O}_{\mathcal{F}}(h):\left(f, \alpha_{*}\right) \mapsto\left(g, \beta_{*}\right)$. Then the total weight of $\left(f, \alpha_{*}\right)$ is greater than or equal to that of $\left(g, \beta_{*}\right)$. Further, the total weights of $\left(f, \alpha_{*}\right)$ and $\left(g, \beta_{*}\right)$ are equal if and only if for all $j$ such that $h\left(s_{j}\right)$ is the basepoint of $T$, we have $i_{j}=0$.

Proof. Let $S^{\prime}=h_{+}^{-1} T$ and let $\overline{S^{\prime}}$ be the points of $S$ which map to the basepoint of $T$. Since $S=S^{\prime} \vee \overline{S^{\prime}}$, we have $f(S) \cong f\left(S^{\prime}\right) \otimes f\left(\bar{S}^{\prime}\right)$, and since $g(T)=f\left(S^{\prime}\right)$, we have $f(S) \cong g(T) \otimes f\left(\bar{S}^{\prime}\right)$. Therefore the total weight of $\left(f, \alpha_{*}\right)$ is greater than or equal to that of $\left(g, \beta_{*}\right)$. Further, the total weights of $\left(f, \alpha_{*}\right)$ and $\left(g, \beta_{*}\right)$ are equal if and only if $f\left(\bar{S}^{\prime}\right)$ is the identity object of $\mathcal{O}_{\mathcal{F}}$. Equivalently, for all $s_{j} \in \bar{S}^{\prime}, f\left(\left\{s_{j}\right\}\right)$ is the identity object, and hence $i_{j}=0$.

Next we discuss the above definitions in the context of the Barratt-Eccles construction to give them a geometric interpretation. If $X$ is a simplicial set we write $X^{n}$ for the $n$-fold Cartesian product and $X^{(n)}$ for the $n$-fold smash product. Recall that Barratt and Eccles [B-E] define a simplicial monoid $\Gamma^{+}(X)$, whose group completion has the homotopy type of $Q X=\Omega^{\infty} \Sigma^{\infty} X$, by

$$
\Gamma^{+}(X)=\coprod_{n \in \mathbf{Z}_{+}} E \Sigma_{n} \times \Sigma_{n} X^{n} / \sim .
$$

The quotient takes points of $E \Sigma_{n} \times \Sigma_{n} X^{n}$ that have any coordinate at the basepoint of $X$ and identifies them with points in $E \Sigma_{n-1} \times_{\Sigma_{n-1}} X^{n-1}$. Hence $\Gamma^{+}(X)$ is filtered by $n$, with the $n$th filtration being

$$
\coprod_{i \leq n} E \Sigma_{i} \times \Sigma_{i} X^{i} / \sim
$$

and with $n$th filtration quotient $\left(E \Sigma_{n}\right)_{+} \wedge_{\Sigma_{n}} X^{(n)}$. If $X$ is $(j-1)$-connected, then one can show that these filtration quotients are at least $(2 j-1)$-connected for $n>1$, 
and hence $H_{i}\left(\Gamma^{+} X, X\right)=0$ for $i<2 j$. This leads to the conclusion that if $X$ is connected, then $\Gamma^{+} X$ is weakly equivalent to $X$ in the stable range, an important step in showing that $\Gamma^{+} X \simeq Q X$.

If we apply $\Gamma^{+}$to a finite pointed set $S$, then $\Gamma^{+}(S)$ is actually the disjoint union of the filtration quotients:

$$
\Gamma^{+}(S) \simeq \coprod_{n}\left(E \Sigma_{n}\right)_{+} \wedge_{\Sigma_{n}} S^{(n)} .
$$

If $k$ is the number of non-basepoint elements of $S$, the component corresponding to a point of $S^{(n)}$ where each coordinate $s_{j}$ appears $i_{j}$ times has the form

$$
E \Sigma_{n} /\left(\Sigma_{i_{1}} \times \ldots \times \Sigma_{i_{k}}\right) \simeq B \Sigma_{i_{1}} \times \ldots \times B \Sigma_{i_{k}}
$$

and so we see that

$$
\Gamma^{+}(S) \simeq\left(\coprod_{i} B \Sigma_{i}\right)^{k}
$$

This is required in order for $\Gamma^{+}(-)$actually to give a $\Gamma$-space in the sense of Segal, because it must take wedges to products and $S$ is a wedge of $k$ copies of $S^{0}$, while $\Gamma^{+}\left(S^{0}\right)=\coprod_{i} B \Sigma_{i}$. Note that the $n$th filtration quotient of $\Gamma^{+}(S)$ is homotopy equivalent to

$$
\coprod_{i_{1}+\ldots+i_{k}=n} B \Sigma_{i_{1}} \times \ldots \times B \Sigma_{i_{k}} .
$$

The simplicial set $\left|\mathcal{O}_{\mathcal{F}}(S)\right|$ is analogous to the Barratt-Eccles construction. Although there is no simple combinatorial expression for $\left|\mathcal{O}_{\mathcal{F}}(S)\right|$ like that for $\Gamma^{+}(S)$, it is true that if $S$ is a finite pointed set with $k$ nonbasepoint elements, then

$$
\left|\mathcal{O}_{\mathcal{F}}(S)\right| \simeq\left(\coprod_{i} B \mathcal{F}_{i}\right)^{k}
$$

In fact, the component of $\mathcal{O}_{\mathcal{F}}(S)$ consisting of objects of type $\left(i_{1}, \ldots, i_{k}\right)$ gives the component $B \mathcal{F}_{i_{1}} \times \ldots \times B \mathcal{F}_{i_{k}}$ in $\left|\mathcal{O}_{\mathcal{F}}(S)\right|$. Because the objects of $\mathcal{O}_{\mathcal{F}}(S)$ of total weight $n$ have type $\left(i_{1}, \ldots, i_{k}\right)$ where $i_{1}+\ldots+i_{k}=n$, the weight filtration on $\left|\mathcal{O}_{\mathcal{F}}(S)\right|$ has the property that the $n$th filtration quotient is homotopy equivalent to

$$
\coprod_{i_{1}+\ldots+i_{k}=n} B \mathcal{F}_{i_{1}} \times \ldots \times B \mathcal{F}_{i_{k}} .
$$

It is natural to ask whether the component $B \mathcal{F}_{i_{1}} \times \ldots \times B \mathcal{F}_{i_{k}}$ is homotopy equivalent to $E \mathcal{F}_{n} /\left(\Sigma_{i_{1}} \times \ldots \times \Sigma_{i_{k}}\right)$, so that the filtration quotients would have an expression similar to that for the quotients in the Barratt-Eccles construction.

The answer in general is no, because in contrast to the fact that $E \Sigma_{n}$ is an acceptable model for $E\left(\Sigma_{i_{1}} \times \ldots \times \Sigma_{i_{k}}\right)$, it is not true that $E \mathcal{F}_{n}$ has the correct fixed point properties to be $E\left(\mathcal{F}_{i_{1}} \times \ldots \times \mathcal{F}_{i_{k}}\right)$. However, in the presence of the product projection condition on the families, the answer is yes, as stated below in Corollary 3.6. The main technical issue in the proof of Theorem 1.1] is to show that the weak equivalence of Corollary 3.6 can be made natural and hence prolongs to simplicial sets. This is the content of Proposition 4.8, whose proof constitutes Section 4 Once this is established, it is possible to analyze the spectrum $A$ associated to a choice of families by looking at the weight filtration on $\left|\mathcal{O}_{\mathcal{F}}\left(S^{j}\right)\right|$, the 
$j$ th space in the spectrum, and using the connectivity of the filtration quotients $\left(E \mathcal{F}_{n}\right)_{+} \wedge_{\Sigma_{n}}\left(S^{j}\right)^{(n)}$ to throw away the parts that are not in the stable range.

Lemma 3.4. Let $\mathcal{F}$ be a compatible collection of families satisfying the product projection condition. Then $E \mathcal{F}_{n}$ is $\Sigma_{i_{1}} \times \ldots \times \Sigma_{i_{k}}$-equivariantly homotopy equivalent to $E \mathcal{F}_{i_{1}} \times \ldots \times E \mathcal{F}_{i_{k}}$.

Proof. It is sufficient to show that $E \mathcal{F}_{n}$ has the correct fixed point property with respect to $\mathcal{F}_{i_{1}} \times \ldots \times \mathcal{F}_{i_{k}}$, the product family of subgroups of $\Sigma_{i_{1}} \times \ldots \times \Sigma_{i_{k}}$. If $H$ is a subgroup of $\Sigma_{i_{1}} \times \ldots \times \Sigma_{i_{k}}$ and $H \notin \mathcal{F}_{i_{1}} \times \ldots \times \mathcal{F}_{i_{k}}$, then by the product projection condition on the families we know $H \notin \mathcal{F}_{n}$ and $\left(E \mathcal{F}_{n}\right)^{H} \simeq \emptyset$. On the other hand, the compatibility condition on the families tells us that if $H \in \mathcal{F}_{i_{1}} \times \ldots \times \mathcal{F}_{i_{k}}$ then $H \in \mathcal{F}_{n}$, hence $\left(E \mathcal{F}_{n}\right)^{H} \simeq *$.

Corollary 3.5. Let $\mathcal{F}$ be a compatible collection of families satisfying the product projection condition. Then

$$
E \mathcal{F}_{n} /\left(\Sigma_{i_{1}} \times \ldots \times \Sigma_{i_{k}}\right) \simeq B \mathcal{F}_{i_{1}} \times \ldots \times B \mathcal{F}_{i_{k}} .
$$

Corollary 3.6. If $S$ is a finite pointed set and $\mathcal{F}$ is a compatible collection of families which satisfies the product projection condition, then the nth filtration quotient of the weight filtration on $\left|\mathcal{O}_{\mathcal{F}}(S)\right|$ is homotopy equivalent to

$$
\left(E \mathcal{F}_{n}\right)_{+} \wedge_{\Sigma_{n}} S^{(n)} .
$$

Proof. We already known that the $n$th filtration quotient is homotopy equivalent to $\coprod_{i_{1}+\ldots+i_{k}=n} B \mathcal{F}_{i_{1}} \times \ldots \times B \mathcal{F}_{i_{k}}$. Let $s_{i_{1}, \ldots, i_{k}}$ be the point of $S^{(n)}$ whose first $i_{1}$ coordinates are $s_{1}$, whose second $i_{2}$ coordinates are $s_{2}$, and so on. Then $\left(E \mathcal{F}_{n}\right) \wedge_{\Sigma_{n}}$ $s_{i_{1}, \ldots, i_{k}} \simeq\left(E \mathcal{F}_{n}\right) /\left(\Sigma_{i_{1}} \times \ldots \times \Sigma_{i_{k}}\right) \simeq B \mathcal{F}_{i_{1}} \times \ldots \times B \mathcal{F}_{i_{k}}$.

Corollary 3.6 indicates the importance of Borel-type constructions in our context, and our last item for this section is to give an expression for a space of this form as the nerve of a category. This will be useful in constructing weak equivalences between the filtration quotients and other spaces which are expressed as the nerves of categories.

Let $T$ be a finite pointed $\Sigma_{n}$-set. Let $\mathcal{O}\left(\mathcal{F}_{n} ; T\right)$ be the category whose objects are pairs $(O,(x, t))$ where $O \in \mathcal{O}_{\mathcal{F}_{n}}$ and $(x, t) \in O \times_{\Sigma_{n}} T$, with the identification that all objects whose $T$-coordinate is the basepoint are identified to a single object whose only endomorphism is the identity. There are morphisms $(O,(x, t)) \rightarrow\left(O^{\prime},\left(x^{\prime}, t^{\prime}\right)\right)$ only if $t=t^{\prime}$, in which case the morphisms are $\Sigma_{n}$-equivariant maps $O \rightarrow O^{\prime}$ which take $x$ to $x^{\prime}$.

Lemma 3.7. Let $T$ be a finite pointed $\Sigma_{n}$-set. Then

$$
\left|\mathcal{O}\left(\mathcal{F}_{n} ; T\right)\right| \cong\left(E \mathcal{F}_{n}\right)_{+} \wedge_{\Sigma_{n}} T \text {. }
$$

Proof. The space $E \mathcal{F}_{n}$ is the nerve of the category whose objects are pairs $(O, x)$ where $O$ is a $\Sigma_{n}$-orbit with $\mathcal{F}_{n}$-isotropy and $x \in O$, and whose morphisms are $\Sigma_{n}$-equivariant maps preserving the distinguished point (see Section 2). Thus we can regard $E \mathcal{F}_{n} \times T$ as the nerve of the category whose objects are triples $(O, x, t)$, where $t \in T$. There are morphisms $(O, x, t) \rightarrow\left(O^{\prime}, x^{\prime}, t^{\prime}\right)$ only if $t=t^{\prime}$, and then the morphisms are equivariant maps $O \rightarrow O^{\prime}$ such that $x \mapsto x^{\prime}$. To take the quotient of $E \mathcal{F}_{n} \times T$ by the $\Sigma_{n}$-action, we take the quotient of the $\Sigma_{n}$-action on the category, that is, we regard $(x, t)$ as an element of $O \times_{\Sigma_{n}} T$. Finally, to obtain $\left(E \mathcal{F}_{n}\right)_{+} \wedge_{\Sigma_{n}} T$ from $E \mathcal{F}_{n} \times_{\Sigma_{n}} T$, we must quotient out $E \mathcal{F}_{n} \times *$. We accomplish this on the level of 
the category by identifying together all objects $(O,(x, t))$ where $t$ is the basepoint of $T$.

\section{Filtration Quotients of the Weight filtration}

Let $X$ be a pointed simplicial set which is finite in each dimension and fix a positive integer $n$. The goal of this section is Proposition 4.8, which asserts that if the product projection condition holds, then the filtration quotient $W_{n} / W_{n-1}\left|\mathcal{O}_{\mathcal{F}}(X)\right|$ of the weight filtration is weakly equivalent to $\left(E \mathcal{F}_{n}\right)_{+} \wedge_{\Sigma_{n}} X^{(n)}$. We already know by Corollary 3.6 that if $S$ is a finite pointed set, then $W_{n} / W_{n-1}\left|\mathcal{O}_{\mathcal{F}}(S)\right|$ and $\left(E \mathcal{F}_{n}\right)_{+} \wedge_{\Sigma_{n}} S^{(n)}$ are in fact homotopy equivalent. The work of this section is to make that equivalence natural in $S$, so that it will prolong to a weak equivalence when $S$ is replaced by a pointed simplicial set $X$.

The ideal would be to obtain the desired equivalence as a natural transformation of functors on finite pointed sets,

$$
W_{n} / W_{n-1} \mathcal{O}_{\mathcal{F}}(S) \rightarrow \mathcal{O}\left(\mathcal{F}_{n} ; S^{(n)}\right)
$$

the latter being a category whose nerve is, by Lemma 3.7 homotopy equivalent to $\left(E \mathcal{F}_{n}\right)_{+} \wedge_{\Sigma_{n}} S^{(n)}$. Unfortunately, we were not able to do this, and instead we take the following more circuitous approach. We define a subcategory $\overline{\mathcal{O}_{\mathcal{F}}(S)}$ of $\mathcal{O}_{\mathcal{F}}(S)$ such that $\overline{\mathcal{O}_{\mathcal{F}}(S)} \hookrightarrow \mathcal{O}_{\mathcal{F}}(S)$ is an equivalence of categories. Then we define a functor

$$
P: W_{n} / W_{n-1} \overline{\mathcal{O}_{\mathcal{F}}(S)} \rightarrow \mathcal{O}\left(\mathcal{F}_{n} ; S^{(n)}\right)
$$

which is natural in $S$ and gives a homotopy equivalence on nerves if the product projection condition holds.

Given an object $\left(f, \alpha_{*}\right)$ of $\mathcal{O}_{\mathcal{F}}(S)$, let $S_{f} \subseteq S$ be the basepoint together with points $s \in S$ such that $f(\{s\})$ is not the identity element of $\mathcal{O}_{\mathcal{F}}(S)$.

Definition 4.1. Let $\overline{\mathcal{O}_{\mathcal{F}}(S)}$ be the full subcategory of $\mathcal{O}_{\mathcal{F}}(S)$ whose objects $\left(f, \alpha_{*}\right)$ satisfy the condition that for every $S^{\prime} \subseteq S$, we have $f\left(S^{\prime}\right)=f\left(S^{\prime} \cap S_{f}\right)$.

Lemma 4.2. The inclusion $I: \overline{\mathcal{O}_{\mathcal{F}}(S)} \rightarrow \mathcal{O}_{\mathcal{F}}(S)$ is an equivalence of categories.

Proof. We define a retraction $R: \mathcal{O}_{\mathcal{F}}(S) \rightarrow \overline{\mathcal{O}_{\mathcal{F}}(S)}$ by letting $R\left(f, \alpha_{*}\right)=\left(\bar{f}, \bar{\alpha}_{*}\right)$, where $\bar{f}\left(S^{\prime}\right)=f\left(S^{\prime} \cap S_{f}\right)$ and $\bar{\alpha}_{S_{1}, S_{2}}=\alpha_{S_{1} \cap S_{f}, S_{2} \cap S_{f}}$. The composite $R I$ is the identity on $\overline{\mathcal{O}_{\mathcal{F}}(S)}$. We need a natural transformation from $I R$ to the identity on $\mathcal{O}_{\mathcal{F}}(S)$. Suppose that $I R\left(f, \alpha_{*}\right)=\left(\bar{f}, \bar{\alpha}_{*}\right)$. Let + denote the identity object of $\mathcal{O}_{\mathcal{F}}$ and let $S_{f}^{c}$ be the pointed complement of $S_{f}$ in $S$, that is, the basepoint and the nonbasepoint elements of $S$ which are not in $S_{f}$. To define a morphism $\left(\bar{f}, \bar{\alpha}_{*}\right) \rightarrow\left(f, \alpha_{*}\right)$ we must give maps $\bar{f}\left(S^{\prime}\right) \rightarrow f\left(S^{\prime}\right)$ in $\mathcal{O}_{\mathcal{F}}$ for each $S^{\prime} \subseteq S$ and commuting diagrams involving the collections $\alpha_{*}$ and $\bar{\alpha}_{*}$. For the first, we use

$$
\begin{aligned}
\bar{f}\left(S^{\prime}\right) & \equiv f\left(S^{\prime} \cap S_{f}\right) \\
& \cong f\left(S^{\prime} \cap S_{f}\right) \otimes+ \\
& =f\left(S^{\prime} \cap S_{f}\right) \otimes f\left(S^{\prime} \cap S_{f}^{c}\right) \\
& \rightarrow f\left(S^{\prime}\right),
\end{aligned}
$$


where the last map is given by $\alpha_{S^{\prime} \cap S_{f}, S^{\prime} \cap S_{f}^{c}}$. For the second, we note that commutativity of the diagrams

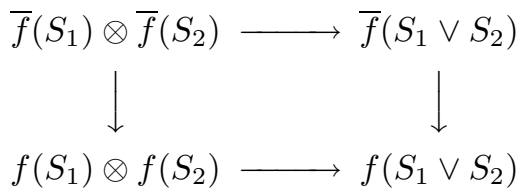

is guaranteed by the coherence of the maps in $\alpha_{*}$.

The category $\overline{\mathcal{O}_{\mathcal{F}}(S)}$ has a conspicuous failing: it is not natural in $S$. The problem is that although a map $h: S \rightarrow T$ of finite pointed sets induces a functor $\mathcal{O}_{\mathcal{F}}(h): \mathcal{O}_{\mathcal{F}}(S) \rightarrow \mathcal{O}_{\mathcal{F}}(T)$, that functor may not carry $\overline{\mathcal{O}_{\mathcal{F}}(S)}$ to $\overline{\mathcal{O}_{\mathcal{F}}(T)}$. However, $\overline{\mathcal{O}_{\mathcal{F}}(S)}$ inherits a weight filtration from $\mathcal{O}_{\mathcal{F}}(S)$, and the filtration quotients are natural.

Lemma 4.3. $W_{n} / W_{n-1} \overline{\mathcal{O}_{\mathcal{F}}(-)}$ is a functor from finite pointed sets to categories.

Proof. Let $h: S \rightarrow T$ be a map of finite pointed sets and suppose that $\left(f, \alpha_{*}\right)$ is an object of $\overline{\mathcal{O}_{\mathcal{F}}(S)}$ of weight $n$. If $\mathcal{O}_{\mathcal{F}}(h):\left(f, \alpha_{*}\right) \mapsto\left(g, \beta_{*}\right)$, then we must show that either the weight of $\left(g, \beta_{*}\right)$ is strictly less than $n$ or else $\left(g, \beta_{*}\right)$ satisfies the condition to be in $\overline{\mathcal{O}_{\mathcal{F}}(T)}$, namely $g\left(T^{\prime}\right)=g\left(T^{\prime} \cap T_{g}\right)$ for every $T^{\prime} \subseteq T$. If $\left(f, \alpha_{*}\right)$ and $\left(g, \beta_{*}\right)$ have equal weights, then we claim that by Lemma $3.3, S_{f} \subseteq h_{+}^{-1}(T)$, for if $s_{j} \in S_{f}$ then $i_{j} \neq 0$, and therefore $h\left(\left\{s_{j}\right\}\right)$ is a nonbasepoint element of $T$. Thus we have

$$
\begin{aligned}
g\left(T^{\prime} \cap T_{g}\right) & \equiv f\left(h_{+}^{-1}\left(T^{\prime} \cap T_{g}\right)\right) \\
& =f\left(h_{+}^{-1} T^{\prime} \cap h_{+}^{-1} T_{g}\right) \\
& =f\left(h_{+}^{-1} T^{\prime} \cap h_{+}^{-1} T_{g} \cap S_{f}\right) \\
& =f\left(h_{+}^{-1} T^{\prime} \cap S_{f}\right) \\
& =f\left(h_{+}^{-1} T^{\prime}\right) \\
& =g\left(T^{\prime}\right),
\end{aligned}
$$

where the third and the next to last equalities come from the fact that $\left(f, \alpha_{*}\right) \in$ $\operatorname{Obj}\left(\overline{\mathcal{O}_{\mathcal{F}}(S)}\right)$.

Remark 4.4. The retraction $R: \mathcal{O}_{\mathcal{F}}(S) \rightarrow \overline{\mathcal{O}_{\mathcal{F}}(S)}$ has no chance to be natural in $S$, because the category $\overline{\mathcal{O}_{\mathcal{F}}(S)}$ is not natural in $S$. However, even on the associated graded, $R: W_{n} / W_{n-1} \mathcal{O}_{\mathcal{F}}(S) \rightarrow W_{n} / W_{n-1} \overline{\mathcal{O}_{\mathcal{F}}(S)}$ fails to be natural. For suppose the diagram

$$
\begin{gathered}
W_{n} / W_{n-1} \mathcal{O}_{\mathcal{F}}(S) \stackrel{R}{\longrightarrow} W_{n} / W_{n-1} \overline{\mathcal{O}_{\mathcal{F}}(S)} \\
\mathcal{O}_{\mathcal{F}}(h) \downarrow \\
W_{n} / W_{n-1} \mathcal{O}_{\mathcal{F}}(T) \stackrel{R}{\longrightarrow} W_{n} / W_{n-1} \overline{\mathcal{O}_{\mathcal{F}}(T)}
\end{gathered}
$$

were commutative, and that following an element $\left(f, \alpha_{*}\right)$ around gave

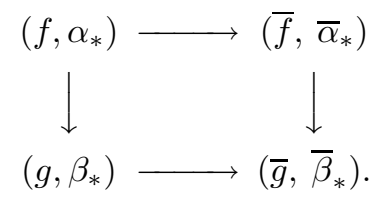


On the one hand, $\bar{g}\left(T^{\prime}\right)=g\left(T^{\prime} \cap T_{g}\right)=f\left(h_{+}^{-1} T^{\prime} \cap h_{+}^{-1} T_{g}\right)$. On the other hand, $\bar{g}\left(T^{\prime}\right)=\bar{f}\left(h_{+}^{-1} T^{\prime}\right)=f\left(h_{+}^{-1} T^{\prime} \cap S_{f}\right)$. However, it is quite possible for $h_{+}^{-1} T_{g}$ to be different from $S_{f}$, even if $\left(f, \alpha_{*}\right)$ and $\left(g, \beta_{*}\right)$ have the same weight, and hence these two calculations of $\bar{g}\left(T^{\prime}\right)$ need not agree. Nevertheless, there is a natural transformation from $\mathcal{O}_{\mathcal{F}}(h) \circ R$ to $R \circ \overline{\mathcal{O}_{\mathcal{F}}(h)}$, and so once we pass to nerves, the diagram

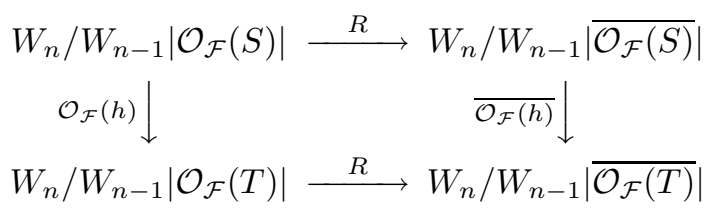

commutes up to homotopy.

Now we are ready to define the functor $P: W_{n} / W_{n-1} \overline{\mathcal{O}_{\mathcal{F}}(S)} \rightarrow \mathcal{O}\left(\mathcal{F}_{n} ; S^{(n)}\right)$. Recall that the objects of the target category are pairs $(O,(x, \bar{s}))$, where $O \in \mathcal{O}_{\mathcal{F}_{n}}$ and $(x, \bar{s}) \in O \times_{\Sigma_{n}} S^{(n)}$, where all objects with $\bar{s}$ equal to the basepoint are identified together to a single basepoint object. The functor $P$ on the equivalence class $W_{n-1} \overline{\mathcal{O}_{\mathcal{F}}(S)}$ is, of course, this basepoint object of $\mathcal{O}\left(\mathcal{F}_{n} ; S^{(n)}\right)$.

To define $P$ on an object $\left(f, \alpha_{*}\right)$ of $W_{n} \overline{\mathcal{O}_{\mathcal{F}}(S)}$ which has weight $n$, let $S=$ $\left\{s_{1}, \ldots, s_{k}\right\}$ be an enumeration of the nonbasepoint elements of $S$. (As in Section 3 we assume that the basepoint is present in any subset of $S$, even though it is not specifically listed.) To define $P$, we must give a $\Sigma_{n}$-orbit, a distinguished point in the orbit, and a point in $S^{(n)}$. The $\Sigma_{n}$-orbit is $f(S)$. To define the distinguished point $y \in f(S)$, let $O_{j}=f\left(\left\{s_{j}\right\}\right)$ and choose a distinguished point $x_{j} \in O_{j}$ for each $j$. Let $e$ denote the identity element of $\Sigma_{n}$. Then we define $y \in f(S)$ as the image of $\left(e,\left(\ldots\left(e, x_{1}, x_{2}\right), \ldots\right), x_{k}\right)$ under the composite of maps from $\alpha_{*}$

$$
\left(\ldots\left(\left(O_{1} \otimes O_{2}\right) \otimes O_{3}\right) \otimes \ldots\right) \otimes O_{k} \rightarrow f(S) .
$$

Finally, let $s_{i_{1}, \ldots, i_{k}} \in S^{(n)}$ be the point whose first $i_{1}$ coordinates are $s_{1}$, whose second $i_{2}$ coordinates are $s_{2}$, and so on. Then we define

$$
P\left(f, \alpha_{*}\right)=\left(f(S),\left(y, s_{i_{1}, \ldots, i_{k}}\right)\right) .
$$

Lemma 4.5. $P$ is well-defined.

Proof. We must show that $P$ does not depend on the choice of the elements $x_{j}$ or on the enumeration of $S$.

Suppose we make another choice of distinguished points, say $x_{j}^{\prime}=\sigma_{j} x_{j}$ for some $\sigma_{j} \in \Sigma_{i_{j}}$. Certainly the orbit $f(S)$ and the point $s_{i_{1}, \ldots, i_{k}}$ do not depend on the choices of elements $x_{j}$. If we let $y^{\prime}$ be the distinguished element of $f(S)$ obtained by using the distinguished points $x_{j}^{\prime}$, then it is easy to check that $y^{\prime}=\left(\sigma_{1} \times \ldots \times \sigma_{k}\right) y$. Since $\sigma_{1} \times \ldots \times \sigma_{k}$ fixes $s_{i_{1}, \ldots, i_{k}}$, the pairs $\left(y^{\prime}, s_{i_{1}, \ldots, i_{k}}\right)$ and $\left(y, s_{i_{1}, \ldots, i_{k}}\right)$ represent the same element of $f(S) \times \Sigma_{n} S^{(n)}$.

To show that $P$ does not depend on the enumeration of $S$, let $\left\{s_{1}, \ldots, s_{k}\right\}$ and $\left\{s_{1}^{\prime}, \ldots, s_{k}^{\prime}\right\}$ be enumerations of the nonbasepoint elements of $S$ which give rise, respectively, to the points $\left(y, s_{i_{1}, \ldots, i_{k}}\right)$ and $\left(y^{\prime}, s_{i_{1}^{\prime}, \ldots, i_{k}^{\prime}}^{\prime}\right)$ of $f(S) \times_{\Sigma_{n}} S^{(n)}$. Let $\sigma \in \Sigma_{k}$ be such that $\left(s_{1}^{\prime}, \ldots, s_{k}^{\prime}\right)=\sigma\left(s_{1}, \ldots, s_{k}\right) \equiv\left(s_{\sigma^{-1}(1)}, \ldots, s_{\sigma^{-1}(k)}\right)$, and let $\bar{\sigma} \in \Sigma_{n}$ be the corresponding block permutation for blocks of size $i_{1}, \ldots, i_{k}$, which has the property that

$$
s_{i_{1}^{\prime}, \ldots, i_{k}^{\prime}}^{\prime}=\bar{\sigma} s_{i_{1}, \ldots, i_{k}} .
$$


We will show that we can take $y^{\prime}$ to be $\bar{\sigma} y$, so that $\left(y, s_{i_{1}, \ldots, i_{k}}\right)$ and $\left(y^{\prime}, s_{i_{1}^{\prime}, \ldots, i_{k}^{\prime}}^{\prime}\right)$ represent the same element of $f(S) \times \Sigma_{n} S^{(n)}$, thus concluding the proof of the lemma.

Recall that $O_{j}=f\left(\left\{s_{j}\right\}\right)$, and let $O_{j}^{\prime}=f\left(\left\{s_{j}^{\prime}\right\}\right)$. Hence $O_{j}^{\prime}=O_{\sigma^{-1}(j)}$, since $s_{j}^{\prime}=s_{\sigma^{-1}(j)}$. For each $j$, choose $x_{j} \in O_{j}$ and let $x_{j}^{\prime}=x_{\sigma^{-1}(j)}$, so that we are looking at the same distinguished elements of the primed and unprimed orbits. Consider how the element $\left(e,\left(\ldots\left(e, x_{1}, x_{2}\right), \ldots\right), x_{k}\right)$ maps from the upper left corner around the commuting diagram

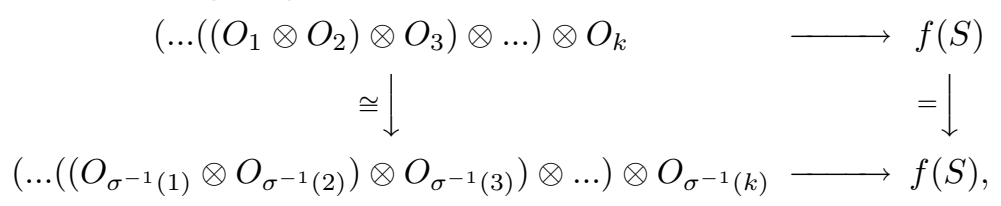

where the left vertical map is given by the associativity and commutativity isomorphisms of the symmetric monoidal category $\mathcal{O}_{\mathcal{F}}$ and the horizontal maps are given by composites from $\alpha_{*}$. Going first to the right and then down, we get $y$. Going down and then to the right, we have

$$
\begin{aligned}
\left(e,\left(\ldots\left(e, x_{1}, x_{2}\right), \ldots\right), x_{k}\right) & \mapsto\left(\bar{\sigma}^{-1},\left(\ldots\left(e, x_{\sigma^{-1}(1)}, x_{\sigma^{-1}(2)}\right), \ldots\right), x_{\sigma^{-1}(k)}\right) \\
& =\bar{\sigma}^{-1}\left(e,\left(\ldots\left(e, x_{1}^{\prime}, x_{2}^{\prime}\right), \ldots\right), x_{k}^{\prime}\right) \\
& =\bar{\sigma}^{-1} y^{\prime} .
\end{aligned}
$$

Hence $y=\bar{\sigma}^{-1} y^{\prime}$, finishing the proof of the lemma.

We will be interested in the map $W_{n} / W_{n-1}\left|\overline{\mathcal{O}_{\mathcal{F}}(S)}\right| \rightarrow\left(E \mathcal{F}_{n}\right)_{+} \wedge_{\Sigma_{n}} S^{(n)}$ which $P$ induces on nerves. Since we actually want to study $W_{n} / W_{n-1}\left|\mathcal{O}_{\mathcal{F}}(X)\right|$ for a simplicial set $X$, it is critical that $P$ be natural so that we can prolong to simplicial sets. That is the content of the following proposition.

Proposition 4.6. $P$ is a natural transformation of functors of finite pointed sets.

Proof. Suppose we have a map $h: S \rightarrow T$ of pointed sets. We must check commutativity of the diagram

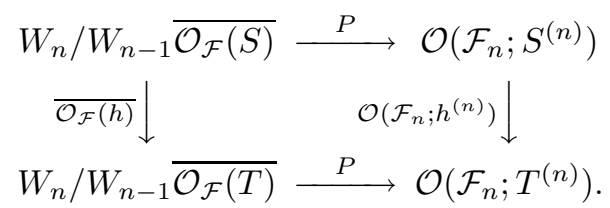

Certainly the diagram commutes on the basepoint component. Suppose we follow an element $\left(f, \alpha_{*}\right)$ of type $\left(i_{1}, \ldots, i_{k}\right)$ and total weight $n$ partway around the diagram to obtain

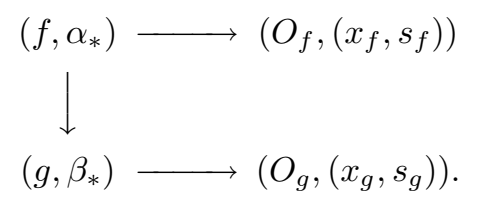

To prove the lemma, we must show $\mathcal{O}\left(\mathcal{F}_{n} ; h^{(n)}\right):\left(O_{f},\left(x_{f}, s_{f}\right)\right) \mapsto\left(O_{g},\left(x_{g}, s_{g}\right)\right)$. By Lemma 3.3 there are two cases to consider.

1. The total weights of $\left(f, \alpha_{*}\right)$ and $\left(g, \beta_{*}\right)$ are equal.

In this case, for all $j$ such that $h\left(s_{j}\right)$ is the basepoint of $T$, we have $i_{j}=0$. We first check that $O_{g}=O_{f}$. By definition, $O_{g}=g(T)=f\left(h_{+}^{-1}(T)\right)=$ 
$f\left(h_{+}^{-1}(T) \cap S_{f}\right)$, where the last equality comes from the fact that $\left(f, \alpha_{*}\right) \in$ $\operatorname{Obj}\left(\overline{\mathcal{O}_{\mathcal{F}}(S)}\right)$. As in the proof of Lemma $4.3 S_{f} \subseteq h_{+}^{-1}(T)$. Hence

$$
O_{g}=f\left(h_{+}^{-1}(T) \cap S_{f}\right)=f\left(S_{f}\right)=f\left(S \cap S_{f}\right)=f(S)=O_{f} .
$$

Next we assume that the enumerations of $S$ and $T$ are chosen so that $h$ is order preserving, and this makes it easy to check that $h^{(n)} s_{f}=t_{g}$. Lastly, we address the distinguished point and show how to choose the distinguished point in $g\left(\left\{t_{1}\right\}\right)$, the others being defined similarly. Suppose that $s_{1}, \ldots, s_{l}$ are the elements of $S$ which map to $t_{1}$ and suppose that $x_{1} \in f\left(\left\{s_{1}\right\}\right), \ldots, x_{l} \in$ $f\left(\left\{s_{l}\right\}\right)$ are the distinguished points chosen to define $P\left(f, \alpha_{*}\right)$. Then we take the distinguished point in $g\left(\left\{t_{1}\right\}\right) \equiv f\left(\left\{s_{1}, \ldots, s_{l}\right\}\right)$ to be the image of $\left(e,\left(\ldots\left(e, x_{1}, x_{2}\right), \ldots\right), x_{l}\right)$ under the appropriate composite of maps from $\alpha_{*}$

$$
\left(\ldots\left(\left(O_{1} \otimes O_{2}\right) \otimes O_{3}\right) \otimes \ldots\right) \otimes O_{l} \rightarrow f\left(\left\{s_{1}, \ldots, s_{l}\right\}\right) .
$$

Then the fact that $x_{f}=x_{g}$ is implied by the associativity isomorphisms of $\mathcal{O}_{\mathcal{F}}$, which are respected by the maps in $\alpha_{*}$.

2. The total weight of $\left(g, \beta_{*}\right)$ is strictly less than that of $\left(f, \alpha_{*}\right)$.

In this case, $\left(g, \beta_{*}\right) \in W_{n-1} \overline{\mathcal{O}_{\mathcal{F}}(T)}$, and hence $\left(O_{g},\left(x_{g}, s_{g}\right)\right)$ is the basepoint object. We claim that $\mathcal{O}\left(\mathcal{F}_{n} ; h^{(n)}\right)$ maps $\left(O_{f},\left(x_{f}, s_{f}\right)\right)$ to the basepoint object of $\mathcal{O}\left(\mathcal{F}_{n} ; T^{(n)}\right)$ because $h^{(n)}\left(s_{f}\right)$ is the basepoint of $T^{(n)}$. This happens because for some $j$, we have that $h\left(s_{j}\right)$ is the basepoint of $T$ but $i_{j}>0$. Since $s_{f}$ has $i_{j}$ coordinates which are $s_{j}$ and we are mapping into a smash product, $h^{(n)}\left(s_{f}\right)$ is the basepoint.

The next proposition is the technical heart of this section.

Proposition 4.7. Let $\mathcal{F}$ be a compatible collection of families satisfying the product projection condition. Then the functor $P$ induces a map on nerves

$$
|P|:\left|W_{n} / W_{n-1} \overline{\mathcal{O}_{\mathcal{F}}(S)}\right| \rightarrow\left(E \mathcal{F}_{n}\right)_{+} \wedge_{\Sigma_{n}} S^{(n)}
$$

which is a natural homotopy equivalence.

Proof. We will give two functors $P^{\prime}$ and $P^{\prime \prime}$ such that $P^{\prime} \circ P^{\prime \prime}: W_{n} / W_{n-1} \overline{\mathcal{O}_{\mathcal{F}}(S)} \rightarrow$ $\mathcal{O}\left(\mathcal{F}_{n} ; S^{(n)}\right)$ and such that there is a natural transformation from $P^{\prime} \circ P^{\prime \prime}$ to $P$. The proposition then follows from showing that $P^{\prime}$ and $P^{\prime \prime}$ induce homotopy equivalences on nerves. This brings up the question of why we need the functor $P$ in the first place, when $P^{\prime} \circ P^{\prime \prime}$ induces the desired homotopy equivalence, and the answer is that the functor $P^{\prime} \circ P^{\prime \prime}$ is not natural in maps of finite pointed sets and so would not prolong to simplicial sets to give Proposition 4.8.

If $S$ is a finite pointed set with enumeration $S=\left\{s_{1}, \ldots, s_{k}\right\}$, let $\mathcal{O}_{\mathcal{F}}(S) \rightarrow\left(\mathcal{O}_{\mathcal{F}}\right)^{k}$ be the functor which takes $\left(f, \alpha_{*}\right)$ to $f\left(\left\{s_{1}\right\}\right) \times \ldots \times f\left(\left\{s_{k}\right\}\right)$, and define $P^{\prime \prime}$ by the composite

$$
P^{\prime \prime}: \overline{\mathcal{O}_{\mathcal{F}}(S)} \hookrightarrow \mathcal{O}_{\mathcal{F}}(S) \rightarrow\left(\mathcal{O}_{\mathcal{F}}\right)^{k} .
$$

By Lemma 4.2 and the fact that $S \mapsto \mathcal{O}_{\mathcal{F}}(S)$ is a $\Gamma$-category, $P^{\prime \prime}$ induces an equivalence of categories. Further, we can filter $\left(\mathcal{O}_{\mathcal{F}}\right)^{k}$ by letting $W_{n}\left(\mathcal{O}_{\mathcal{F}}\right)^{k}$ be the disjoint union of the components $\mathcal{O}_{\mathcal{F}_{i_{1}}} \times \ldots \times \mathcal{O}_{\mathcal{F}_{i_{k}}}$ with $i_{1}+\ldots+i_{k} \leq n$. Then $P^{\prime \prime}$ is filtration preserving and gives equivalences on the filtration quotients

$$
P^{\prime \prime}: W_{n} / W_{n-1} \overline{\mathcal{O}_{\mathcal{F}}(S)} \rightarrow W_{n} / W_{n-1}\left(\mathcal{O}_{\mathcal{F}}\right)^{k}
$$


We define the functor $P^{\prime}: W_{n} / W_{n-1}\left(\mathcal{O}_{\mathcal{F}}\right)^{k} \rightarrow \mathcal{O}\left(\mathcal{F}_{n} ; S^{(n)}\right)$ as a quotient. First we need two new categories and some facts about them.

1. Let $\mathcal{E}_{\mathcal{F}}$ be the category whose objects are pairs $(O, x)$ where $O$ is an object of $\mathcal{O}_{\mathcal{F}}$ and $x \in O$, and whose morphisms are equivariant maps which preserve the distinguished point. There is a weight filtration on $\left(\mathcal{E}_{\mathcal{F}}\right)^{k}$ similar to that of $\left(\mathcal{O}_{\mathcal{F}}\right)^{k}$, and the filtration is preserved by the projection $\left(\mathcal{E}_{\mathcal{F}}\right)^{k} \rightarrow\left(\mathcal{O}_{\mathcal{F}}\right)^{k}$ which forgets the distinguished point. We define the $\left(i_{1}, \ldots, i_{k}\right)$-component of $\left(\mathcal{E}_{\mathcal{F}}\right)^{k}$ to be the component which maps to $\mathcal{O}_{\mathcal{F}_{i_{1}}} \times \ldots \times \mathcal{O}_{\mathcal{F}_{i_{k}}}$, and note that $\Sigma_{i_{1}} \times \ldots \times \Sigma_{i_{k}}$ acts on this component by acting on the distinguished points. Fixed point properties show readily that the nerve of the $\left(i_{1}, \ldots, i_{k}\right)$-component of $\left(\mathcal{E}_{\mathcal{F}}\right)^{k}$ is $E \mathcal{F}_{i_{1}} \times \ldots \times E \mathcal{F}_{i_{k}}$.

2. Let $\mathcal{E}\left(\mathcal{F}_{n} ; S^{(n)}\right)$ be the category whose objects are pairs $(O,(x, \bar{s}))$, where $O$ is a $\Sigma_{n}$-orbit and $(x, \bar{s}) \in O \times S^{(n)}$ (rather than $O \times \Sigma_{n} S^{(n)}$, as in the category $\left.\mathcal{O}\left(\mathcal{F}_{n} ; S^{(n)}\right)\right)$. All objects whose $S^{(n)}$-coordinate is the basepoint are identified to a single basepoint object with only the identity endomorphism. The set of morphisms $(O,(x, \bar{s})) \rightarrow\left(O^{\prime},\left(x^{\prime}, \bar{s}^{\prime}\right)\right)$ is empty unless $\bar{s}=\bar{s}^{\prime}$, in which case the morphisms are equivariant maps $O \rightarrow O^{\prime}$ which take $x$ to $x^{\prime}$. Each component of the nerve is isomorphic to $E \mathcal{F}_{n}$. The group $\Sigma_{n}$ acts on the category by acting on the point $(x, \bar{s})$, and the component of objects whose $S^{(n)}$-coordinate is $s_{i_{1}, \ldots, i_{k}}$ is invariant under the action of $\Sigma_{i_{1}} \times \ldots \times \Sigma_{i_{k}} \subseteq \Sigma_{n}$.

Define a functor $\overline{P^{\prime}}: W_{n} / W_{n-1}\left(\mathcal{E}_{\mathcal{F}}\right)^{k} \rightarrow \mathcal{E}\left(\mathcal{F}_{n} ; S^{(n)}\right)$ which maps the $\left(i_{1}, \ldots, i_{k}\right)$ component of $\left(\mathcal{E}_{\mathcal{F}}\right)^{k}$ to the $s_{i_{1}, \ldots, i_{k}}$-component of $\mathcal{E}\left(\mathcal{F}_{n} ; S^{(n)}\right)$ by

$$
\overline{P^{\prime}}\left(\left(O_{1}, x_{1}\right), \ldots,\left(O_{k}, x_{k}\right)\right)=\left(O,\left(x, s_{i_{1}, \ldots, i_{k}}\right)\right),
$$

where

$$
\begin{gathered}
O=\left(\ldots\left(\left(O_{1} \otimes O_{2}\right) \otimes O_{3}\right) \ldots\right) \otimes O_{k}, \\
x=\left(e,\left(\ldots\left(e, x_{1}, x_{2}\right) \ldots\right), x_{k}\right) .
\end{gathered}
$$

Since $\overline{P^{\prime}}$ is $\Sigma_{i_{1}} \times \ldots \times \Sigma_{i_{k}}$-equivariant on the $\left(i_{1}, \ldots, i_{k}\right)$-component, Lemma 3.4 and Proposition 2.2(1) tell us that $\overline{P^{\prime}}$ is a $\Sigma_{i_{1}} \times \ldots \times \Sigma_{i_{k}}$-equivariant homotopy equivalence on that component.

Now define $P^{\prime}$ by taking $\Sigma_{i_{1}} \times \ldots \times \Sigma_{i_{k}}$-orbits on the various components:

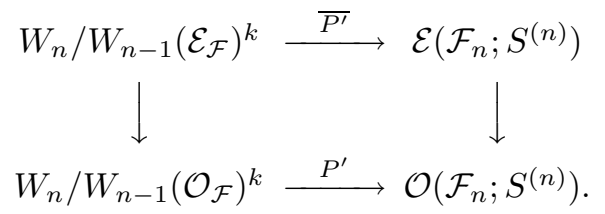

Because $\overline{P^{\prime}}$ gives equivariant homotopy equivalences, $P^{\prime}$ is a homotopy equivalence. Further, it is easy to check that there is a natural transformation $\left(P^{\prime} \circ P^{\prime \prime}\right) \rightarrow P$, finishing the proof of the lemma.

Proposition 4.8. Let $X$ be a simplicial set and let $\mathcal{F}$ be a compatible collection of families satisfying the product projection condition. Then

$$
W_{n} / W_{n-1}\left|\mathcal{O}_{\mathcal{F}}(X)\right| \simeq\left(E \mathcal{F}_{n}\right)_{+} \wedge_{\Sigma_{n}}(X)^{(n)} .
$$

Proof. The functors

$$
W_{n} / W_{n-1} \mathcal{O}_{\mathcal{F}}(S) \leftarrow W_{n} / W_{n-1} \overline{\mathcal{O}_{\mathcal{F}}(S)} \rightarrow \mathcal{O}\left(\mathcal{F}_{n} ; S^{(n)}\right)
$$


are natural in maps of $S$ and induce homotopy equivalences on nerves

$$
\left|W_{n} / W_{n-1} \mathcal{O}_{\mathcal{F}}(S)\right| \leftarrow\left|W_{n} / W_{n-1} \overline{\mathcal{O}_{\mathcal{F}}(S)}\right| \rightarrow\left(E \mathcal{F}_{n}\right)_{+} \wedge_{\Sigma_{n}}(S)^{(n)}
$$

Naturality in $S$ allows us to prolong to simplicial sets.

\section{THE $n$ TH FAMILY INCLUSION MAP}

In this section we construct the $n$th family inclusion map that appears twice in the pushout diagram of Theorem 1.1 We will show how it interacts with the weight filtration, a detail we will need in Section 6. We then give an alternate construction and use this version to discuss the $n$th family inclusion map in the example of stable homotopy, where it turns out to be related to the transfer.

Given a compatible collection of families $\mathcal{F}$ with associated spectrum $A$, the $n$th family inclusion map will be a map of spectra

$$
I_{\mathcal{F}_{n}}: \Sigma^{\infty}\left(B \mathcal{F}_{n}\right)_{+} \rightarrow A
$$

which by construction will be natural in the choice of families. It will turn out to be the same as the map obtained by taking the inclusion $\left(B \mathcal{F}_{n}\right)_{+} \hookrightarrow \coprod_{i} B \mathcal{F}_{i}$, group completing on the right to obtain an infinite loop space, and extending over $\Sigma^{\infty}\left(B \mathcal{F}_{n}\right)_{+}$. Let $S$ be a finite pointed set. We begin by constructing a map

$$
\left(B \mathcal{F}_{n}\right)_{+} \wedge S \rightarrow\left|\mathcal{O}_{\mathcal{F}}(S)\right|
$$

by giving $S$ the trivial $\Sigma_{n}$-action, constructing a functor

$$
I_{\mathcal{F}_{n}}(S): \mathcal{O}\left(\mathcal{F}_{n} ; S\right) \rightarrow \mathcal{O}_{\mathcal{F}}(S),
$$

and taking nerves. By Lemma $3.7\left(B \mathcal{F}_{n}\right)_{+} \wedge S$ is the nerve of the category $\mathcal{O}\left(\mathcal{F}_{n} ; S\right)$, whose objects are pairs $(O,(x, s))$ for $O \in \operatorname{Obj}\left(\mathcal{O}_{\mathcal{F}_{n}}\right)$ and $(x, s) \in O \times_{\Sigma_{n}} S$. Since $S$ has the trivial $\Sigma_{n}$-action, these objects can be regarded simply as pairs $(O, s)$. Hence to define the map $I_{\mathcal{F}_{n}}(S)$, it is sufficient to assign to a pair $(O, s)$ an object $\left(f, \alpha_{*}\right) \in \operatorname{Obj}\left(\mathcal{O}_{\mathcal{F}}(S)\right)$. To define the function $f: P(S) \rightarrow \mathcal{O}_{\mathcal{F}}$ on a pointed subset $S^{\prime} \subseteq S$, we let $f\left(S^{\prime}\right)=O$ if $s$ is a nonbasepoint element of $S^{\prime}$ and $f\left(S^{\prime}\right)=+$ otherwise, where + denotes the identity object in $\mathcal{O}_{\mathcal{F}}$. All of the isomorphisms in the collection $\alpha_{*}$ are identity maps. Note that $I_{\mathcal{F}_{n}}(S)$ actually has its image in the subcategory $W_{n} \overline{\mathcal{O}_{\mathcal{F}}(S)}$.

Lemma 5.1. Let $\mathcal{F}$ be a compatible collection of families and let $S$ be a finite pointed set. For each $n$, there is a commuting diagram

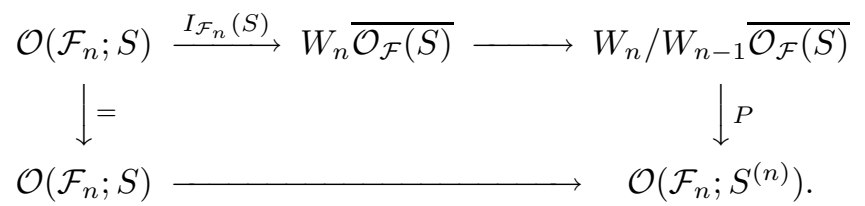

where the lower horizontal map is induced by the diagonal map $S \rightarrow S^{(n)}$.

Proof. The proof is an easy verification using the definitions of $I_{\mathcal{F}_{n}}(S)$ and $P$.

Since $I_{\mathcal{F}_{n}}(S)$ is natural in $S$, we can prolong the preceding lemma to simplicial sets and take nerves to obtain the following corollary, which will be needed in Section 6. 
Corollary 5.2. Let $X$ be a simplicial set. For each n, there is a commuting diagram

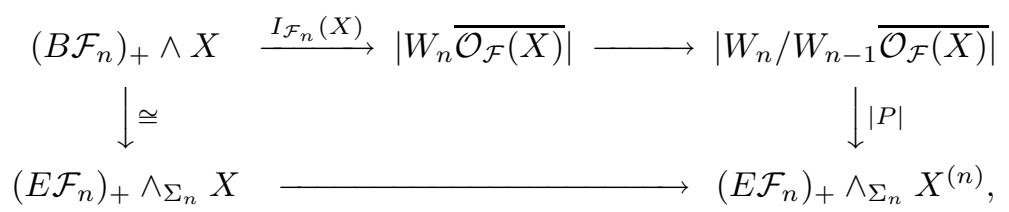

where the lower horizontal map is induced by the diagonal map $X \rightarrow X^{(n)}$.

To define the map of spectra

$$
I_{\mathcal{F}_{n}}: \Sigma^{\infty}\left(B \mathcal{F}_{n}\right)_{+} \rightarrow A,
$$

we use the maps

$$
I_{\mathcal{F}_{n}}\left(S^{j}\right):\left(B \mathcal{F}_{n}\right)_{+} \wedge S^{j} \rightarrow\left|\mathcal{O}_{\mathcal{F}}\left(S^{j}\right)\right|
$$

between the $j$ th spaces of the spectra. To show that these maps are compatible under suspension, we will need the following standard construction. Given finite pointed sets $S$ and $T$, there is a map $\left|\mathcal{O}_{\mathcal{F}}(S)\right| \wedge T \rightarrow\left|\mathcal{O}_{\mathcal{F}}(S \wedge T)\right|$ which sends $\left|\mathcal{O}_{\mathcal{F}}(S)\right| \wedge t$ by the functor $\left|\mathcal{O}_{\mathcal{F}}(-)\right|$ applied to the map $S \rightarrow S \wedge t \rightarrow S \wedge T$. The structure maps of $A$ are prolongations

$$
\left|\mathcal{O}_{\mathcal{F}}\left(S^{j}\right)\right| \wedge S^{1} \rightarrow\left|\mathcal{O}_{\mathcal{F}}\left(S^{j} \wedge S^{1}\right)\right|
$$

of this standard construction to the simplicial set $S^{1}$. (See, for example, B-F, Section 4].) Hence compatibility under suspension can be verified by checking the commutativity of the diagram

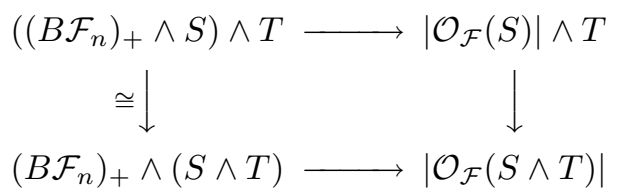

at the level of simplices and prolonging to the simplicial sets $S^{j}$ in place of $S$ and $S^{1}$ in place of $T$. Therefore the maps $I_{\mathcal{F}_{n}}\left(S^{j}\right):\left(B \mathcal{F}_{n}\right)_{+} \wedge S^{j} \rightarrow\left|\mathcal{O}_{\mathcal{F}}\left(S^{j}\right)\right|$ fit together to give a map of spectra

$$
I_{\mathcal{F}_{n}}: \Sigma^{\infty}\left(B \mathcal{F}_{n}\right)_{+} \rightarrow A .
$$

Remark 5.3.

1. It is easy to verify from the definition that $I_{\mathcal{F}_{n}}\left(S^{0}\right):\left(B \mathcal{F}_{n}\right)_{+} \rightarrow \mathcal{O}_{\mathcal{F}}\left(S^{0}\right)$ is the inclusion $\left(B \mathcal{F}_{n}\right)_{+} \hookrightarrow \coprod_{i} B \mathcal{F}_{i}$. Hence the map $I_{\mathcal{F}_{n}}: \Sigma^{\infty}\left(B \mathcal{F}_{n}\right)_{+} \rightarrow A$ is the same as the map obtained by taking the inclusion $\left(B \mathcal{F}_{n}\right)_{+} \hookrightarrow \coprod_{i} B \mathcal{F}_{i}$, group completing on the right to obtain an infinite loop space, and extending over $\Sigma^{\infty}\left(B \mathcal{F}_{n}\right)_{+}$.

2. As an example, we take $\mathcal{F}$ to be the family of trivial subgroups $\{e\} \subseteq \Sigma_{n}$. In this case, $A \simeq S^{0}$, and $I_{\mathcal{F}_{n}}$ gives a map of spectra

$$
\Sigma^{\infty}\left(B \Sigma_{n}\right)_{+} \rightarrow S^{0} .
$$

By examining the construction of the transfer given in $\mathrm{K}-\mathrm{P}$, one can show that this map of spectra is the composite of the transfer $\Sigma^{\infty}\left(B \Sigma_{n}\right)_{+} \rightarrow$ $\Sigma^{\infty}\left(B \Sigma_{n-1}\right)_{+}$with the augmentation $\Sigma^{\infty}\left(B \Sigma_{n-1}\right)_{+} \rightarrow S^{0}$. We will return to this example in Section [7. 


\section{Proof of Theorem 1.1}

In this section we give the proof of Theorem 1.1 whose statement we reproduce for the reader's convenience.

Theorem [1.1. Let $\mathcal{F}$ be a compatible collection of families that satisfies the product projection condition. Let $A$ be the associated spectrum, and let $A_{m}$ denote the $m$ th filtration of $A$ by complexity. Let $\mathcal{F}_{m}^{\prime}$ be the family of subgroups in $\mathcal{F}_{m}$ which do not act transitively on $\{1, \ldots, m\}$. Then there is a stable homotopy pushout diagram,

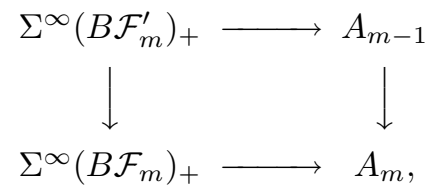

where the vertical maps are given by inclusions of the $(m-1)$ st complexity filtration in the mth, and the horizontal maps are given by mth family inclusion maps. The pushout diagram is natural in $\mathcal{F}$.

The strategy of the proof is to examine the diagram of $j$ th spaces and to show that it is a pushout in the stable range. We emphasize that there are two filtrations at work in this situation. The first is the filtration of the spectrum $A$ by complexity, which is the subject of Theorem 1.1. The second is the weight filtration of Section 3 which is the main tool in the proof of Theorem 1.1. Applying the weight filtration to the spaces which make up the spectra $A_{m-1}$ and $A_{m}$ and considering the connectivity of the filtration quotients will enable us to concentrate on the diagram in just the stable range.

To construct the commuting diagram of Theorem 1.1 fix a compatible collection of families $\mathcal{F}$ which satisfies the product projection condition. The complexity filtration defined in Section 2 gives us two new collections of families, $C_{m-1} \mathcal{F}$ and $C_{m} \mathcal{F}$, each of which is compatible and satisfies the product projection condition, and which have associated spectra $A_{m-1}$ and $A_{m}$, respectively. Naturality of the $m$ th family inclusion map gives us a commuting diagram

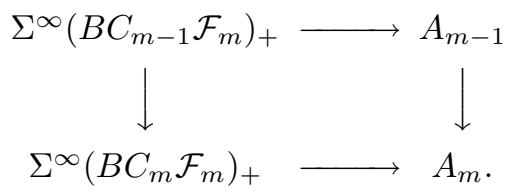

Note that $C_{m} \mathcal{F}_{m}=\mathcal{F}_{m}$, and $C_{m-1} \mathcal{F}_{m}$ is precisely the subgroups in $\mathcal{F}_{m}$ which do not act transitively on $\{1, \ldots, m\}$, so $C_{m-1} \mathcal{F}_{m}=\mathcal{F}_{m}^{\prime}$. Hence we obtain the diagram of Theorem 1.1

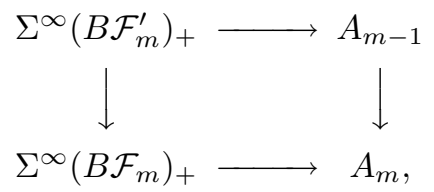

which we are asserting is a homotopy pushout diagram.

We will be looking at this square diagram of spectra at the level of the $j$ th spaces. Let the $j$ th space of $A_{m-1}$ be $Y_{j}=\left|\mathcal{O}_{C_{m-1}} \mathcal{F}\left(S^{j}\right)\right|$ and let the $j$ th space of $A_{m}$ be $Z_{j}=\left|\mathcal{O}_{C_{m} \mathcal{F}}\left(S^{j}\right)\right|$. To prove the theorem, it is sufficient to show that for each $j$, 
the diagram

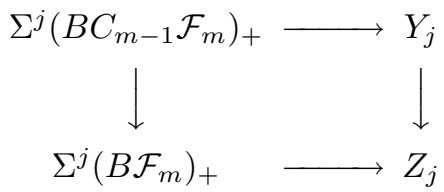

is a homotopy pushout square in the stable range. In fact, it is sufficient to consider $j>1$, and then we can choose a model of $S^{j}$ with no nondegenerate one-simplices. It follows that $Y_{j}$ and $Z_{j}$ likewise have no nondegenerate one-simplices, and thus all the spaces in the diagram are simply connected. (We note that we are relying here on [B-F Cor. 4.10], which tells us that we can use any model of $S^{j}$ that suits our purposes.)

The proof of Theorem 1.1 comes in two parts. First we show that diagram (6.1) is an actual homotopy pushout when we replace $Y_{j}$ and $Z_{j}$ by their $m$ th weight filtrations. Then we show that everything of higher filtration in $Y_{j}$ and $Z_{j}$ is out of the stable range.

Lemma 6.1. If $j>1$, then

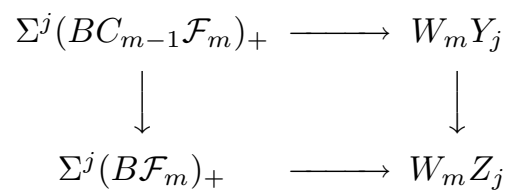

is a homotopy pushout square.

Proof. Since all the spaces in the diagram are simply connected, it is sufficient to show that the cofibers of the vertical maps are weakly equivalent. We approach the question by the following $2 \times 3$ diagram of simply connected spaces:

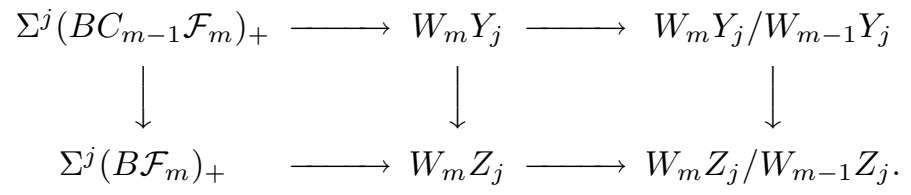

By examining the sequence of vertical cofibers, it is easy to show that if the right hand square and the outer square are homotopy pushouts, then so is the left square, which is what we are trying to prove.

To see that we have a homotopy pushout square on the right, we show that $W_{m-1} Y_{j} \rightarrow W_{m-1} Z_{j}$ is a weak equivalence. It is sufficient to see that the induced maps on the filtration quotients of the weight filtration are weak equivalences. By Proposition 4.8 the induced maps have the form

$$
\left(E C_{m-1} \mathcal{F}_{n}\right)_{+} \wedge_{\Sigma_{n}}\left(S^{j}\right)^{(n)} \rightarrow\left(E C_{m} \mathcal{F}_{n}\right)_{+} \wedge_{\Sigma_{n}}\left(S^{j}\right)^{(n)},
$$

where $n \leq m-1$. However, if $n \leq m-1$, then $C_{m-1} \mathcal{F}_{n}=\mathcal{F}_{n}=C_{m} \mathcal{F}_{n}$, and hence the map on filtration quotients is a weak equivalence.

On the other hand, by Corollary 5.2 the outside square is equivalent to

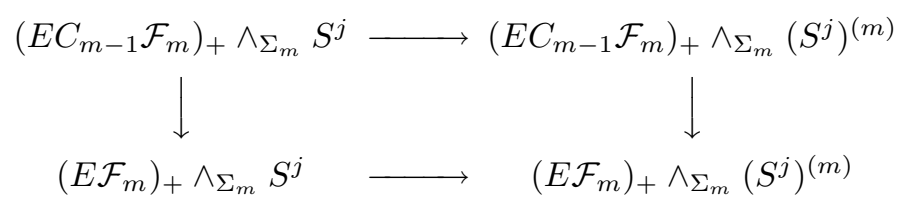


where the horizontal maps are induced by the diagonal. To show that this is a homotopy pushout, we will show that the cofibers of the horizontal maps are homotopy equivalent. Let $\Delta$ denote the diagonal of $\left(S^{j}\right)^{(m)}$ and let $X$ denote $\left(S^{j}\right)^{(m)} / \Delta$. Taking cofibers horizontally in diagram (6.2), we are claiming that $\left(E C_{m-1} \mathcal{F}_{m}\right)_{+} \wedge_{\Sigma_{m}} X \rightarrow\left(E \mathcal{F}_{m}\right)_{+} \wedge_{\Sigma_{m}} X$ is an equivalence. It suffices to show that $\left(E C_{m-1} \mathcal{F}_{m}\right)_{+} \wedge X \rightarrow\left(E \mathcal{F}_{m}\right)_{+} \wedge X$ induces equivalences on the fixed point sets of the action of subgroups $H \subseteq \Sigma_{m}$. There are three cases.

1. If $H \notin \mathcal{F}_{m}$, then $H \notin C_{m-1} \mathcal{F}_{m}$, and $\left(E \mathcal{F}_{m}\right)^{H}=\left(E C_{m-1} \mathcal{F}_{m}\right)^{H}=\emptyset$.

2. If $H \in \mathcal{F}_{m}$ and $H \in C_{m-1} \mathcal{F}_{m}$, then $\left(E \mathcal{F}_{m}\right)^{H} \simeq\left(E C_{m-1} \mathcal{F}_{m}\right)^{H} \simeq *$.

3. If $H \in \mathcal{F}_{m}$, but $H \notin C_{m-1} \mathcal{F}_{m}$, then $\left(E \mathcal{F}_{m}\right)^{H} \simeq *$ while $\left(E C_{m-1} \mathcal{F}_{m}\right)^{H}=\emptyset$.

In this case, however, $H$ must be transitive, so there are no fixed points of $H$ on $X$ except the basepoint. Hence in this case

$$
\left(\left(E C_{m-1} \mathcal{F}_{m}\right)_{+} \wedge X\right)^{H}=\left(\left(E \mathcal{F}_{m}\right)_{+} \wedge X\right)^{H}=*
$$

What we have shown so far is that

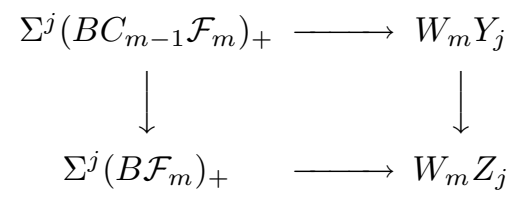

is a homotopy pushout square, and what we want to show, to establish Theorem 1.1 is that

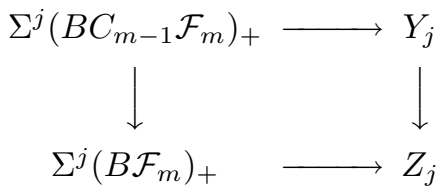

is a homotopy pushout square in the stable range. Hence Theorem 1.1 follows from the next lemma.

Lemma 6.2. $W_{m} Y_{j} \rightarrow Y_{j}$ and $W_{m} Z_{j} \rightarrow Z_{j}$ are weak equivalences in the stable range.

Proof. Since $Y_{j}$ and $Z_{j}$ are $(j-1)$-connected, it is sufficient to show that the quotients $Y_{j} / W_{m} Y_{j}$ and $Z_{j} / W_{m} Z_{j}$ are $(2 j-1)$-connected. We know that they are simply connected, because $Y_{j}$ and $Z_{j}$ have been chosen to have no nondegenerate one-cells. By Proposition 4.8, the weight filtration quotients of both $Y_{j} / W_{m} Y_{j}$ and $Z_{j} / W_{m} Z_{j}$ are of the form $\left(E C_{k} \mathcal{F}_{n}\right)_{+} \wedge_{\Sigma_{n}}\left(S^{j}\right)^{(n)}$, where $k=m-1$ for $Y_{j}$ and $k=m$ for $Z_{j}$, and $n \geq m+1>k$. By the following lemma, these are $(2 j-1)$ connected in homology, and so $Y_{j} / W_{m} Y_{j}$ and $Z_{j} / W_{m} Z_{j}$ are likewise, which implies the lemma.

Lemma 6.3. If $n>k$, then $\bar{H}_{i}\left(E C_{k} \mathcal{F}_{n}\right)_{+} \wedge_{\Sigma_{n}}\left(S^{j}\right)^{(n)}=0$ if $0 \leq i \leq 2 j-1$.

Proof. Let $\mathcal{O}(G)$ be the orbit category of a group $G$. For any $G$-space $Y$ there is a spectral sequence

$$
E_{2}^{p, q}=\underset{\mathcal{O}(G)}{\lim _{p}^{p}} H_{q} \operatorname{map}_{G}(O, Y) \Rightarrow H_{p+q} Y / G
$$


This is the spectral sequence for the homology of a homotopy colimit $\mathrm{B}-\mathrm{K}$ applied to

$$
\operatorname{hocolim}_{\mathcal{O}(G)} \operatorname{map}_{G}(O, Y) \simeq Y / G
$$

(see, for example, [E]). If $O=G / H$, then $\operatorname{map}_{G}(O, Y) \cong Y^{H}$, so the input to the spectral sequence is the homology of the fixed point sets. We apply this spectral sequence with $Y=\left(E C_{k} \mathcal{F}_{n}\right)_{+} \wedge\left(S^{j}\right)^{(n)}$ and $G=\Sigma_{n}$, and so we consider the fixed point sets of subgroups $H \subseteq \Sigma_{n}$ on $\left(E C_{k} \mathcal{F}_{n}\right)_{+} \wedge\left(S^{j}\right)^{(n)}$. There are two cases.

If $H \notin C_{k} \mathcal{F}_{n}$, then $\left(E C_{k} \mathcal{F}_{n}\right)_{+}^{H}$ is just the basepoint. Hence the fixed point set of $H$ on $\left(E C_{k} \mathcal{F}_{n}\right)_{+} \wedge\left(S^{j}\right)^{(n)}$ is likewise just the basepoint, with $H_{0}=\mathbf{Z}$ and $H_{i}=0$ for $i>0$.

If $H \in C_{k} \mathcal{F}_{n}$, then $\left(E C_{k} \mathcal{F}_{n}\right)_{+}^{H} \simeq S^{0}$, because $\left(E C_{k} \mathcal{F}_{n}\right)^{H}$ is contractible. On the other hand, the fixed point set of $H$ on $\left(S^{j}\right)^{(n)}$ is a smash product of copies of $S^{j}$, one for each orbit of $H$ on $\{1, \ldots, n\}$. Because $n>k$ and $H \in C_{k} \mathcal{F}_{n}, H$ cannot act transitively on $\{1, \ldots, n\}$ and must have more than one orbit. Hence

$$
\left(\left(E C_{k} \mathcal{F}_{n}\right)_{+} \wedge\left(S^{j}\right)^{(n)}\right)^{H} \simeq S^{0} \wedge S^{j} \wedge \ldots \wedge S^{j},
$$

where there are at least two factors of $S^{j}$, and so this fixed point set is $(2 j-1)$ connected: $H_{0}=\mathbf{Z}$ and $H_{i}=0$ for $0<i<2 j$.

Now consider the spectral sequence in homology. Since $\mathcal{O}\left(\Sigma_{n}\right)$ has a terminal object, we see that $\mathrm{lim}^{p} H_{0}=0$ for $p>0$ and $\varliminf^{0} H_{0}=\mathbf{Z}$. On the other hand, above dimension 0 , the homology of the fixed point sets appears only in dimensions $2 j$ and higher. Hence $\bar{H}_{*}\left(E C_{k} \mathcal{F}_{n}\right)_{+} \wedge_{\Sigma_{n}}\left(S^{j}\right)^{(n)}$ is $(2 j-1)$-connected.

\section{EXAMPLES}

In this section we give examples of the application of Theorem 1.1 We note the following general principles.

1. The complexity filtration of a spectrum $A$ always starts with $A_{1}=S^{0}$, because only the identity subgroup has complexity 1.

2. The map $A_{m-1} \rightarrow A_{m}$ is a homotopy equivalence unless $\mathcal{F}_{m}^{\prime}$ is different from $\mathcal{F}_{m}$. Hence we need only consider $A_{m-1} \rightarrow A_{m}$ for values of $m$ such that $\mathcal{F}_{m}$ contains transitive subgroups of $\Sigma_{m}$.

3. To compute $A$ at the prime $p$, it is sufficient to consider only the $p$-subgroups in $\mathcal{F}$. This is proved in $[\mathrm{L}$ by a standard transfer argument.

7.1. Elementary abelian $p$-groups of complexity $p$. We first consider the main example of $[\mathrm{L}$, the spectrum associated to the collection of families whose elements are elementary abelian $p$-groups which are generated by products of disjoint $p$ cycles, and subgroups of such groups. Using the complexity terminology, these are the elementary abelian $p$-subgroups of complexity less than or equal to $p$. In $[\mathrm{L}]$ we obtained the following result:

Theorem 7.1 ([L, Theorem 2.3]). Let $\mathcal{F}$ denote the family of elementary abelian $p$-subgroups of complexity less than or equal to $p$. Then, at a prime $q \neq p$, the spectrum $A$ is weakly equivalent to the sphere spectrum, and at the prime $p$, there 
is a homotopy pushout diagram

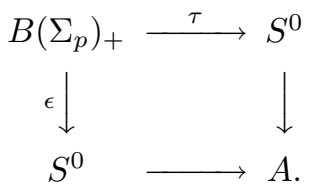

Here $\tau$ denotes the transfer map of $[\mathrm{K}-\mathrm{P}]$ and $\epsilon$ denotes the augmentation.

The proof began by showing that $\coprod_{n} B \mathcal{F}_{n}$ has the homotopy type of a double bar construction

$$
\operatorname{Bar}\left(\coprod B\left(\Sigma_{n} \int \operatorname{Aut}(\mathbf{Z} / p)\right), \coprod B\left(\Sigma_{n} \int N \mathbf{Z} / p\right), \coprod B \Sigma_{n}\right)
$$

where $N \mathbf{Z} / p$ denotes the normalizer of $\mathbf{Z} / p$ in $\Sigma_{p}$. Then we showed that the spectrum associated to the group completion of such a double bar construction is a homotopy pushout of the spectra corresponding to the factors. In our double bar construction, the factor $\coprod B \Sigma_{n}$ corresponds to $S^{0}$, the factor $\coprod B\left(\Sigma_{n} \int \operatorname{Aut}(\mathbf{Z} / p)\right)$ corresponds to $\Sigma^{\infty}(B \operatorname{Aut}(\mathbf{Z} / p))_{+}$, which at the prime $p$ is equivalent to $S^{0}$, and the factor $\coprod B\left(\Sigma_{n} \int N \mathbf{Z} / p\right)$ corresponds to $\Sigma^{\infty}(B N \mathbf{Z} / p)_{+}$, which at the prime $p$ is equivalent to $\Sigma^{\infty}\left(B \Sigma_{p}\right)_{+}$. The proof was lengthy and involved a number of delicate ad hoc calculations.

Using Theorem 1.1 to prove Theorem 7.1 gives a slight strengthening of the result while dramatically shortening the proof. More importantly, it shows how Theorem 7.1 follows from general principles rather than ad hoc methods. We first note that the only value of $m$ for which $\mathcal{F}_{m}$ and $\mathcal{F}_{m}^{\prime}$ differ is $m=p$, because transitive $p$-groups of complexity $p$ can only occur in $\Sigma_{p}$. Hence the spectrum $A$ will be a single homotopy pushout

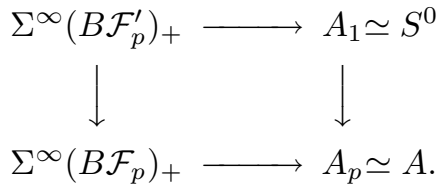

The family $\mathcal{F}_{p}^{\prime}$ consists of elements of $\mathcal{F}_{p}$ which do not act transitively on $\{1, \ldots, p\}$. Hence the only element of $\mathcal{F}_{p}^{\prime}$ is the trivial subgroup, and $B \mathcal{F}_{p}^{\prime} \simeq B \Sigma_{p}$. To compute $B \mathcal{F}_{p}$, we make use of the following lemma.

Lemma 7.2. J-M, Proposition 5.1] Let $\mathcal{C}$ be a category with the following properties:

1. $\mathcal{C}$ has two objects $C_{1}$ and $C_{2}$;

2. $\operatorname{Hom}\left(C_{1}, C_{1}\right)$ is a group $G$ and $\operatorname{Hom}\left(C_{2}, C_{2}\right)$ is a group $H$;

3. $\operatorname{Hom}\left(C_{1}, C_{2}\right)$ is a set $S$ and $\operatorname{Hom}\left(C_{2}, C_{1}\right)$ is empty.

If $F$ is a functor from $\mathcal{C}$ to simplicial sets with $F\left(C_{1}\right)=X_{1}$ and $F\left(C_{2}\right)=X_{2}$, then hocolim $_{\mathcal{C}} F$ is weakly equivalent to the homotopy pushout of the diagram

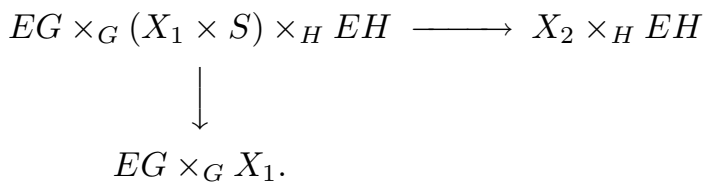

Note that in this lemma, $G$ acts on $X_{1}$ and $S$, while $H$ acts on $X_{2}$ and $S$. 
We can apply the lemma to calculate $B \mathcal{F}_{p}$, which is the nerve of the category whose objects are the free $\Sigma_{p^{-}}$orbit and $\Sigma_{p} /(\mathbf{Z} / p)$, and whose morphisms are $\Sigma_{p^{-}}$ equivariant maps. The automorphism group of the free orbit is, of course, $\Sigma_{p}$ itself, and the set of maps from the free orbit to $\Sigma_{p} /(\mathbf{Z} / p)$ is in one-to-one correspondence with the elements of $\Sigma_{p} /(\mathbf{Z} / p)$. The automorphism group of $\Sigma_{p} /(\mathbf{Z} / p)$ is the quotient of the normalizer of $\mathbf{Z} / p$ by the centralizer of $\mathbf{Z} / p$. Let $C_{p-1}$ be the cyclic group of order $p-1$ acting on $\mathbf{Z} / p$ as its automorphism group. Then $N \mathbf{Z} / p$ is the semidirect product of $\mathbf{Z} / p$ with $C_{p-1}$, and the centralizer of $\mathbf{Z} / p$ is $\mathbf{Z} / p$ itself. Hence the automorphism group of $\Sigma_{p} /(\mathbf{Z} / p)$ is $(N \mathbf{Z} / p) /(\mathbf{Z} / p) \cong C_{p-1}$. We apply the lemma with

$$
\begin{aligned}
& X_{1}=X_{2}=*, \\
& G=\Sigma_{p}, H=C_{p-1}, \\
& S=\Sigma_{p} /(\mathbf{Z} / p),
\end{aligned}
$$

and we obtain a homotopy pushout diagram

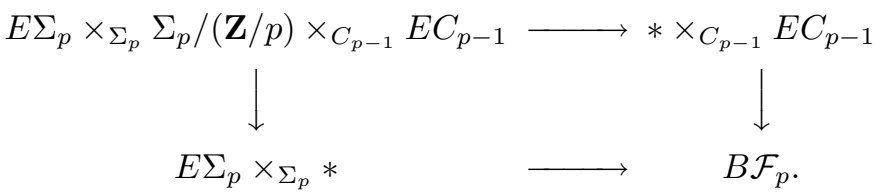

To simplify, note that $E \Sigma_{p} \times_{\Sigma_{p}} \Sigma_{p} /(\mathbf{Z} / p) \simeq B \mathbf{Z} / p$, and then $B \mathbf{Z} / p \times_{C_{p-1}} E C_{p-1} \simeq$ $B N \mathbf{Z} / p$, so the diagram becomes

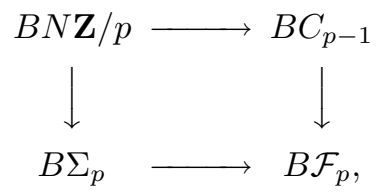

where the top horizontal and the left vertical map are induced by homomorphisms of groups.

Thus we have exhibited the spectrum $A$ as a homotopy pushout

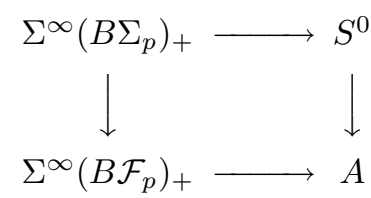

where $B \mathcal{F}_{p}$ is itself given by the homotopy pushout in diagram (7.1). Now we consider whether we recover Theorem 7.1. At a prime $q \neq p, B \Sigma_{p} \rightarrow B \mathcal{F}_{p}$ is a $\bmod q$ homology isomorphism, because in diagram (7.1) we have a mod $q$ homology isomorphism $B N \mathbf{Z} / p \rightarrow B C_{p-1}$. Hence, at $q$ the map $S^{0} \rightarrow A$ is an equivalence. At the prime $p$, on the other hand, $B \mathcal{F}_{p}$ is acyclic, because in diagram (7.1) we have a mod $p$ homology isomorphism $B N \mathbf{Z} / p \rightarrow B \Sigma_{p}$ and $B C_{p-1}$ is acyclic. Thus the vertical map of diagram (7.2), $\Sigma^{\infty}\left(B \Sigma_{p}\right)_{+} \rightarrow \Sigma^{\infty}\left(B \mathcal{F}_{p}\right)_{+}$, is equivalent to the augmentation $\Sigma^{\infty}\left(B \Sigma_{p}\right)_{+} \rightarrow S^{0}$. The horizontal map $\Sigma^{\infty}\left(B \Sigma_{p}\right)_{+} \rightarrow S^{0}$ of diagram (7.2) comes from composing the inclusion $\left(B \Sigma_{p}\right)_{+} \hookrightarrow \bigsqcup_{n} B \Sigma_{n}$ with the map to the group completion of $\coprod_{n} B \Sigma_{n}$, and then extending over $\Sigma^{\infty}\left(B \Sigma_{p}\right)_{+}$. As we discussed at the end of Section [5] on the level of spectra this gives the composite of the transfer map $\Sigma^{\infty}\left(B \Sigma_{p}\right)_{+} \rightarrow \Sigma^{\infty}\left(B \Sigma_{p-1}\right)_{+}$with the augmentation 
$\Sigma^{\infty}\left(B \Sigma_{p-1}\right)_{+} \rightarrow S^{0}$. At the prime $p$, the latter is an equivalence, and hence we have recovered Theorem $[7.1$ with the slight strengthening that we actually have $A$ expressed as a colimit without having to localize at a prime.

7.2. The filtration of $H \mathbf{Z}$ by complexity. In this section we let $\mathcal{F}$ be the family of all subgroups. In Proposition 7.4, we show that the filtration of the associated spectrum by complexity is the filtration of $H \mathbf{Z}$ by symmetric powers of spheres. We obtain Theorem 1.2 as an immediate consequence, and we then recover some results on the symmetric powers of spheres.

The following lemma gives us freedom to manipulate the symmetric monoidal category used to obtain a spectrum.

Lemma 7.3. Let $\mathcal{C} \rightarrow \mathcal{D}$ be a functor between symmetric monoidal categories which respects the symmetric monoidal product and which is an equivalence of categories. Then the induced map between the associated spectra is a weak equivalence.

Proof. Let $S$ be a finite pointed set with $k$ nonbasepoint elements. There is a commuting diagram

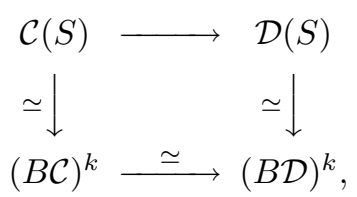

and so $\mathcal{C}(S) \rightarrow \mathcal{D}(S)$ is a homotopy equivalence. Prolongation to simplicial sets tells us that $\mathcal{C}\left(S^{j}\right) \rightarrow \mathcal{D}\left(S^{j}\right)$ is a weak equivalence, and since these are the $j$ th spaces in the spectra associated to $\mathcal{C}$ and $\mathcal{D}$, respectively, we see that the spectra are homotopy equivalent as well.

Proposition 7.4. Let $\mathcal{F}$ be the family of all subgroups. Then the associated spectrum $A$ is the Eilenberg-MacLane spectrum $H \mathbf{Z}$ and the filtration of $A$ by complexity is the filtration of $\mathrm{HZ}$ by the symmetric powers of the sphere spectrum:

$$
A_{m} \simeq S p^{m}\left(S^{0}\right)
$$

Proof. Since $\mathcal{F}$ is the family of all subgroups, each component $\mathcal{O}_{\mathcal{F}_{n}}$ of the symmetric monoidal category $\mathcal{O}_{\mathcal{F}}$ has the trivial $\Sigma_{n}$-orbit as a terminal object. Let $\mathbf{Z}_{+}$be the symmetric monoidal category whose objects are the nonnegative integers, whose morphisms consist only of identity morphisms, and whose symmetric monoidal product is addition of integers. Then the map $\mathcal{O}_{\mathcal{F}} \rightarrow \mathbf{Z}_{+}$which sends all objects of $\mathcal{O}_{\mathcal{F}_{n}}$ to $n$ is an equivalence of categories which respects the symmetric monoidal product, and by Lemma 7.3. $\mathcal{O}_{\mathcal{F}}\left(S^{j}\right) \rightarrow \mathbf{Z}_{+}\left(S^{j}\right)$ is a weak equivalence. The latter is the infinite symmetric power of $S^{j}$, and so $A=H \mathbf{Z}$.

To compute the filtration of $A$ by complexity, recall that $A_{m}$ corresponds to the symmetric monoidal category $\mathcal{O}_{C_{m}} \mathcal{F}$ whose components are the categories $\mathcal{O}_{C_{m}} \mathcal{F}_{n}$ of $\Sigma_{n}$-orbits whose isotropy groups have complexity less than or equal to $m$. If $n \leq m$, then $\mathcal{O}_{C_{m}} \mathcal{F}_{n}=\mathcal{O}_{\mathcal{F}_{n}}$ and so has a terminal object. We define a new category $\overline{\mathcal{O}_{C_{m}} \mathcal{F}}$ as a quotient of $\mathcal{O}_{C_{m} \mathcal{F}}$ : for all $n \leq m$, collapse the component $\mathcal{O}_{C_{m}} \mathcal{F}_{n}$ to a single object with only the identity endomorphism. The category $\overline{\mathcal{O}_{C_{m}} \mathcal{F}}$ looks like $\mathbf{Z}_{+}$up to and including the $m$ th component, looks like $\mathcal{O}_{C_{m}} \mathcal{F}$ thereafter, and has a symmetric monoidal structure. The quotient map $\mathcal{O}_{C_{m}} \mathcal{F} \rightarrow \overline{\mathcal{O}_{C_{m}} \mathcal{F}}$ is an equivalence of categories which respects the symmetric monoidal structure. 
Now consider $\mathcal{O}_{C_{m} \mathcal{F}}\left(S^{j}\right)$, the $j$ th space of $A_{m}$. By Lemma $7.3 \mathcal{O}_{C_{m} \mathcal{F}}\left(S^{j}\right) \rightarrow$ $\overline{\mathcal{O}_{C_{m} \mathcal{F}}}\left(S^{j}\right)$ is a weak equivalence, and we will analyze the latter by using the weight filtration inherited from $\mathcal{O}_{C_{m} \mathcal{F}}\left(S^{j}\right)$. It is easy to see by inspection that $W_{m} \overline{\mathcal{O}_{C_{m} \mathcal{F}}}\left(S^{j}\right) \cong W_{m} \mathbf{Z}_{+} S^{j} \cong S p^{m}\left(S^{j}\right)$. On the other hand, we have a commuting diagram

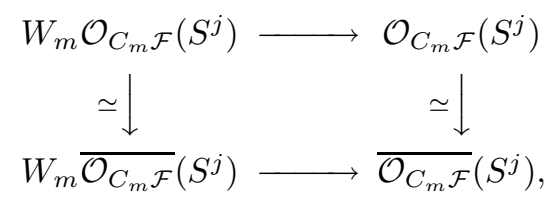

and since the family of all subgroups certainly satisfies the product projection condition, Lemma 6.2 tells us that the top horizontal map is an equivalence in the stable range. The proposition follows.

Proof of Theorem 1.2. Application of Theorem 1.1 gives pushout diagrams

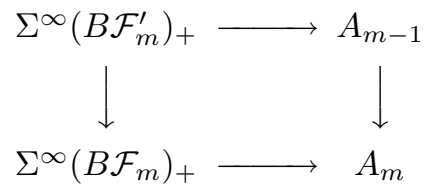

where $\mathcal{F}_{m}$ is the family of all subgroups of $\Sigma_{m}$ and $\mathcal{F}_{m}^{\prime}$ is the family of subgroups of $\Sigma_{m}$ which do not act transitively on $\{1, \ldots, m\}$. Since $B \mathcal{F}_{m}$ is contractible and Proposition 7.4 gives weak equivalences $A_{m-1} \simeq S p^{m-1}\left(S^{0}\right)$ and $A_{m} \simeq S p^{m}\left(S^{0}\right)$, the theorem follows by taking cofibers vertically to get a weak equivalence $S p^{m}\left(S^{0}\right) / S p^{m-1}\left(S^{0}\right) \simeq \Sigma \Sigma^{\infty} B \mathcal{F}_{m}^{\prime}$.

Examples. We offer the following examples of how Theorem 1.2 recovers known facts about symmetric powers of spheres.

1. Consider the equivalence given by Theorem 1.2 in the case $m=2$ :

$$
S p^{2}\left(S^{0}\right) / S p^{1}\left(S^{0}\right) \simeq \Sigma \Sigma^{\infty} B \mathcal{F}_{2}^{\prime} .
$$

The family $\mathcal{F}_{2}^{\prime}$ is the family of nontransitive subgroups of $\Sigma_{2}$, which is to say, only the trivial subgroup. Hence $B \mathcal{F}_{2}^{\prime} \simeq B \Sigma_{2} \simeq R P^{\infty}$, and we recover the result that $S p^{2}\left(S^{0}\right) / S p^{1}\left(S^{0}\right) \simeq \Sigma \Sigma^{\infty} R P^{\infty}$.

2. If $m$ is not a power of $p$, then at the prime $p$ the map $S p^{m-1}\left(S^{0}\right) \rightarrow S p^{m}\left(S^{0}\right)$ is known to be a weak equivalence. To see this from our point of view, we note that if $m$ is not a power of $p$, then the family of nontransitive $p$-subgroups of $\Sigma_{m}$ is the same as the family of all $p$-subgroups of $\Sigma_{m}$, and at the prime $p$ this gives the same classifying space as the family of all subgroups of $\Sigma_{m}$. Hence by the principle mentioned at the beginning of Section $7, B \mathcal{F}_{m}^{\prime}$ is contractible at $p$, and so is $S p^{m}\left(S^{0}\right) / S p^{m-1}\left(S^{0}\right)$.

\section{REFERENCES}

[A] J. F. Adams, "Infinite Loop Spaces", Princeton University Press, Princeton, NJ, 1978. MR 80d:55001

[B-E] M. Barratt and P. Eccles, $\Gamma^{+}$-structures - I: a free group functor for stable homotopy theory, Topology 13 (1974) 25 - 45. MR 50:1234a

[B-F] A. Bousfield and E. Friedlander, Homotopy theory of $\Gamma$-spaces, spectra, and bisimplicial sets, in, "Geometric Applications of Homotopy Theory II", 80-130, M. Barratt and M. Mahowald (eds.), Lecture Notes in Math. 658, Springer-Verlag, 1978. MR 80e:55021 
[B-K] A. Bousfield and D. Kan, Homotopy limits, completions, and localizations, Lecture Notes in Math. 304, Springer-Verlag, 1972. MR 51:1825

[E] A. Elmendorf, Systems of fixed points, Trans. Amer. Math. Soc. 277 (1983) 275-284. MR 84f: 57029

[J-M] S. Jackowski and J. McClure, Homotopy approximations for classifying spaces of compact Lie groups, in, "Algebraic Topology, Proceedings, Arcata, 1986," 221-234, G. Carlsson, R. L. Cohen, H. R. Miller, D. C. Ravenel (eds.), Lecture Notes in Math. 1370, SpringerVerlag, 1989. MR 90m:55012

[K-P] D. Kahn and S. Priddy, The transfer and stable homotopy theory, Math. Proc. Cambridge Philos. Soc. 83 (1978) 103-111. MR 57:4164b

[L] K. Lesh, Infinite loop spaces from group theory, Math. Z. 225 (1997) 467-483. MR 98j:55009

$[\mathrm{M}]$ J. P. May, $E_{\infty}$ spaces, group completions, and permutative categories, in, "New Developments in Topology (Proc. Sympos. Algebraic Topology, Oxford, 1972)," 61-93, London Math. Soc. Lecture Note Series 11, Cambridge Univ. Press, 1974. MR 49:3915

[S] G. Segal, Categories and cohomology theories, Topology 13 (1974) 293-312. MR 50:5782

[tD] T. tom Dieck, Orbittypen und äquivariante homologie. I. Arch. Math. (Basel) 23 (1972) 307-317. MR 46:10017

Department of Mathematics, Univerity of Toledo, Toledo, Ohio 43606-3390

E-mail address: klesh@uoft02.utoledo.edu 